Document downloaded from:

http://hdl.handle.net/10251/52818

This paper must be cited as:

Nadal Soriano, E.; Díez, P.; Ródenas García, JJ.; Tur Valiente, M.; Fuenmayor Fernández, FJ. (2015). A recovery-explicit error estimator in energy norm for linear elasticity. Computer Methods in Applied Mechanics and Engineering. 287:172-190.

doi:10.1016/j.cma.2015.01.013.

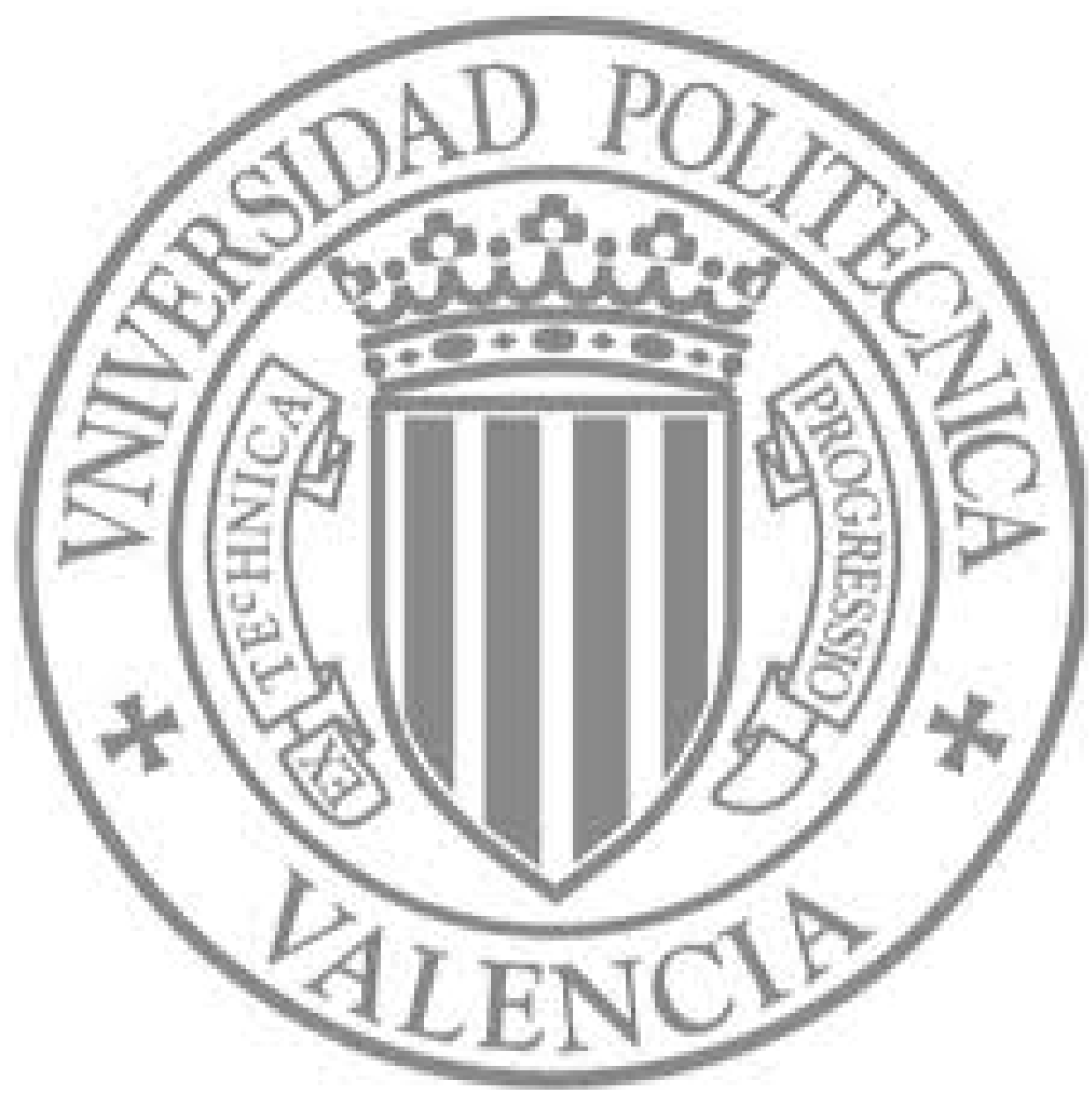

The final publication is available at

http://dx.doi.org/10.1016/j.cma.2015.01.013

Copyright Elsevier 


\title{
A recovery-explicit error estimator in energy norm for linear elasticity
}

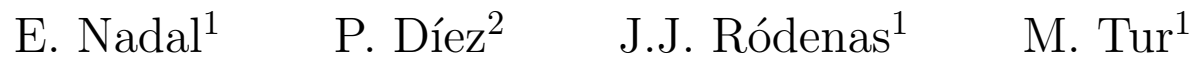 \\ F.J. Fuenmayor ${ }^{1}$
}

${ }^{1}$ Centro de Investigación de Tecnología de Vehículos (CITV),

Universitat Politècnica de València, E-46022-Valencia, Spain, e-mail: jjrodena@mcm.upv.es, ennaso@upv.es, matuva@mcm.upv.es, ffuenmay@mcm.upv.es

${ }^{2}$ LaCaN, Universitat Politècnica de Catalunya \& CIMNE, Barcelona, Spain e-mail: pedro.diez@upc.edu

\begin{abstract}
Significant research effort has been devoted to produce one-sided error estimates for Finite Element Analyses, in particular to provide upper bounds of the actual error. Typically, this has been achieved using residual-type estimates. One of the most popular and simpler (in terms of implementation) techniques used in commercial codes is the recovery-based error estimator. This technique produces accurate estimations of the exact error but is not designed to naturally produce upper bounds of the error in energy norm. Some attempts to remedy this situation provide bounds depending on unknown constants. Here, a new step towards obtaining error bounds from the recoverybased estimates is proposed. The idea is 1) to use a locally equilibrated recovery technique to obtain an accurate estimation of the exact error, 2) to add an explicit-type error bound of the lack of equilibrium of the recovered stresses in order to guarantee a bound of the actual error and 3) to efficiently and accurately evaluate the constants appearing in the bounding expressions, thus providing asymptotic bounds. The the numerical tests with $h$-adaptive refinement process show that the bounding property holds even for coarse meshes, providing upper bounds in practical applications.
\end{abstract}

Error bounding, recovery techniques, explicit residual error estimator

\section{Introduction}

Although the Finite Element Method (FEM) is a powerful method for a vast type of engineering problems, it is well known that, in general, it is only able to provide an approximated solution. Therefore, some error level has to be accounted for to define the safety factors during the design process of structural components. During several years various error estimation techniques have been developed. We can classify the error estimators in three groups based on its convergence through the global effectivity index $\theta$ (ratio of the estimate to the true error): 
- Asymptotically exact: when the richness of the discrete solution space $N$ is increased, the estimated error gets closer (from above or below, or even oscillating) to the true one, then $\theta \rightarrow 1$ when $\operatorname{dim} N \rightarrow \infty$.

- Asymptotically (upper) bounded: when the richness of the discrete solution space is increased, the estimated error provides higher values than the true one, therefore $\theta \geq 1$ when $\operatorname{dim} N \rightarrow \infty$.

- Asymptotically not bounded: when the richness of the discrete solution space is increased, the estimated error provides lower values than the true one, then $\theta \leq 1$ when $\operatorname{dim} N \rightarrow \infty$.

Another way to classify the error estimators is according to the procedure used to obtain the estimates. Traditionally, there are three major branches in the error estimation field: The first group, the residual-based error estimators, introduced by Babuška and Rheinboldt [1] is subdivided into implicit [2, 3, 4] and explicit ones [5]. The explicit ones depend on a constant. Explicit values for the constant have been obtained by several authors [6, 7]. More recently Prof. Stein and coworkers $[8,9]$ have obtained an explicit value which provides highly accurate error estimations for a certain type of problems and elements. The second branch of error estimators, related with the concept of dual analysis, makes use of two solutions, one compatible and one equilibrated. Some of these error estimators solve two global problems in parallel [10] whereas other post-process the FE solution [11, $12,13]$. Under this group we can also include the error estimators based on the Constitutive Relation Error (CRE) introduced by Ladevèze and Leguillon [11] and followed by several contributions for many applications, see for example $[14,15$, 16, 17]. This technique compares a kinematically admissible stress field with a statically admissible solution obtained by solving local problems, built using the strong prolongation condition. These types of error estimators are asymptotically (upper) bounded. Finally, the third branch, the recovery-type error estimators are based on the use of the Zienkiewicz and Zhu (ZZ) error estimator [18]. These techniques were traditionally unable to provide upper bounds of the error in energy norm. The error evaluation is obtained by calculating the energy norm associated with the difference between the FE solution (compatible) and a recovered stress solution (not necessary equilibrated, but continuous), obtained for example with the Superconvergent Patch Recovery (SPR) technique [19, 20]. In this case the recovered stress field is obtained processing the values of the stress field evaluated at superconvergent points. The process produces a recovered solution with higher convergence rate than the theoretical optimum convergence rate of the FE solution. This recovered solution, of a higher accuracy than the FE solution, is introduced in the ZZ error estimator providing accurate results. An intensive analysis of the superconvergent property for different recovery procedures can be found in [21]. References $[22,23,24]$ showed that under certain assumptions, related with the mesh type and regularity of the solution, when the recovered field used for the estimation is obtained with the SPR technique, the error estimator is asymptotically exact. The recovery-based error estimators are robust, easy to implement and are used in commercial codes. The publication of the original SPR technique was followed by 
several works aimed to improve its quality, see for example [25, 26, 27]. Ródenas et al. proposed to add constraints to impose local equilibrium and local compatibility to the recovered solution in the FEM framework [28] bringing up the SPR-C technique that was also adapted to the eXtended Finite Element Method (XFEM) framework $[29,30]$.

Carstensen and Funken [31, 32] presented an error estimator, based on recoverytype error estimators, providing upper bounds of the error in energy norm, under certain assumptions of smoothness of the solution which permit to neglect the lack of internal equilibrium of the recovered solution. Díez et al. [33] presented a methodology to obtain computable upper bounds of the error in the energy norm considering the stress recovered field provided by the SPR-C technique and taking into account the lack of internal equilibrium of the recovered stresses. This technique allowed to obtain one of the firsts procedures to get practical upper bounds for FEM and XFEM based on recovery techniques [33, 34]. Since the SPR-C technique does not provide an equilibrated stress field at the global level, the upper bound property is obtained by adding a correction term to account for equilibrium defaults. So far, only a computationally expensive estimation, based on projection techniques, of these correction terms is available.

In this work we use the Cartesian Grid FEM (cgFEM) presented in $[35,36,37]$ to numerically solve the linear elasticity problem, although, we have to remark that all results presented in this work can be directly extended to the standard FEM. The cgFEM is an immerse boundary method where the geometry is embedded into the mesh domain. Nested meshes are used in cgFEM for $h$-adaptive analysis. Therefore, projection techniques used in the proposed error estimation are efficient and easy-to-implement. In any case, the projections imply a low computational cost as they are only required in the first meshes of the $h$-refinement analysis. Some authors claim that the use of the SPR technique with Cartesian Grids, as the one used in this work, would be problematic. For instance, reference [38] indicates: "Unfortunately, for an implicit mesh it would be very difficult to implement such a superconvergent recovery scheme of the stress field for elements that intersect the boundary". However in the XFEM framework, where the mesh is independent of the crack, efficient recovery techniques have been already proposed based on the Moving Least Squares (MLS) technique [39, 40, 41, 42, 43] and some on the SPR technique $[30,34]$, which introduce worthy improvements to the solution, especially along the boundaries, even in elements trimmed by the crack. In this work we will use the SPR-C technique to obtain an improved stress field from the FE stress field, being this last field rather inaccurate in the case of bilinear elements. The error measure using the ZZ error estimator in combination with the SPR-C technique will guide the $h$-adaptive refinement process. The objective of this work is to present a new technique to evaluate an upper bound of the discretization error in the energy norm for the linear elasticity problem with smooth solution. The technique presented is based on the previously mentioned recovery technique whose use is crucial in the process since the improved field provided by this technique is continuous and fulfils the boundary equilibrium. The main idea of the proposed technique is to obtain an upper bound of the error in the energy norm by evaluating an upper bound of the error correction term introduced in [33] using an explicit-type error evaluation 
technique, that requires the use of a problem-dependent constant. We will also propose a procedure to obtain an accurate evaluation of the constant required by the explicit-type error evaluation technique which allows to obtain error upper bounds in practical application, even for coarse meshes.

The rest of the paper is organized as follows. In section 2 we will set up the elasticity problem and its FE approximation. Section 3 will be devoted to explain the main features of the SPR-C technique. Section 4 will introduce the new bounding procedure and in section 5 we will test the behavior of the proposed technique through some numerical results. Final remarks will be presented in section 6 .

\section{Problem Statement}

In this section we briefly present the model for the 2D linear elasticity problem. We denote the Cauchy stress as $\boldsymbol{\sigma}$, the displacement as $\mathbf{u}$, and the strain as $\boldsymbol{\varepsilon}$, all these fields being defined over the domain $\Omega \subset \mathbb{R}^{2}$, with boundary denoted by $\partial \Omega$. Prescribed tractions denoted by $\mathbf{t}$ are imposed over the part $\Gamma_{N}$ of the boundary, while displacements denoted by $\overline{\mathbf{u}}$ are prescribed over the complementary part $\Gamma_{D}$ of the boundary. Bony loads are denoted as $\mathbf{b}$.

The elasticity problem takes the following form. We seek $(\boldsymbol{\sigma}, \mathbf{u})$ satisfying:

- static admissibility:

$$
\begin{aligned}
\mathbf{L}^{T} \boldsymbol{\sigma}+\mathbf{b} & =\mathbf{0} & & \text { in } \Omega \\
\mathbf{G} \boldsymbol{\sigma} & =\mathbf{t} & & \text { on } \Gamma_{N}
\end{aligned}
$$

where $\mathbf{L}$ is the differential operator defined as:

$$
\mathbf{L}=\left[\begin{array}{cc}
\frac{\partial}{\partial x} & 0 \\
0 & \frac{\partial}{\partial y} \\
\frac{\partial}{\partial y} & \frac{\partial}{\partial x}
\end{array}\right]
$$

and $\mathbf{G}$ is the projection operator that projects the stress field into traction over the boundary. The operator $\mathbf{G}$ is the matrix form of Cauchy's law considering the unit normal $\mathbf{n}=\left\{n_{x} n_{y}\right\}^{T}$ to $\Gamma_{N}$ such that:

$$
\mathbf{G}=\left[\begin{array}{ccc}
n_{x} & 0 & n_{y} \\
0 & n_{y} & n_{x}
\end{array}\right]
$$

- kinematic admissibility:

$$
\mathbf{u}=\overline{\mathbf{u}} \quad \text { on } \Gamma_{D}
$$

- constitutive relation:

$$
\boldsymbol{\sigma}=\mathbf{D} \boldsymbol{\varepsilon}(\mathbf{u}), \text { with } \boldsymbol{\varepsilon}(\mathbf{u})=\mathbf{L u} \quad \text { in } \Omega
$$

where the matrix $\mathbf{D}$ contains the elasticity coefficients of the usual linear isotropic constitutive law relating stress and strain. 
The problem above takes the primal variational form:

$$
\begin{aligned}
& \text { Find } \mathbf{u} \in(V+\{\mathbf{w}\}): \forall \mathbf{v} \in V \\
& a(\mathbf{u}, \mathbf{v})=l(\mathbf{v}) \quad \text { where } \\
& a(\mathbf{u}, \mathbf{v})=\int_{\Omega} \boldsymbol{\varepsilon}(\mathbf{u})^{T} \mathbf{D} \boldsymbol{\varepsilon}(\mathbf{v}) \mathrm{d} \Omega \\
& l(\mathbf{v})=\int_{\Omega} \mathbf{b}^{T} \mathbf{v} \mathrm{d} \Omega+\int_{\Gamma_{N}} \mathbf{t}^{T} \mathbf{v} \mathrm{d} \Gamma
\end{aligned}
$$

where $V=\left\{\mathbf{v}\left|\mathbf{v} \in\left[H^{1}(\Omega)\right]^{2}, \mathbf{v}\right|_{\Gamma_{D}}=\mathbf{0}\right\}$ and $\mathbf{w}$ is a particular displacement field satisfying the Dirichlet boundary conditions.

\subsection{Finite element discretization}

Let us introduce a classical finite element discretization scheme for the elasticity problem. The approximate displacement field $\mathbf{u}^{h}$ is searched for in a space of finite dimension $\left(V^{h}+\{\mathbf{w}\}\right) \subset(V+\{\mathbf{w}\})$ such that $V^{h}$ is spanned by locally supported finite element shape functions.

Using the Galerkin framework, the primal variational formulation (1-6) is recast in the form:

$$
\begin{aligned}
& \text { Find } \mathbf{u}^{h} \in\left(V^{h}+\{\mathbf{w}\}\right): \forall \mathbf{v} \in V^{h} \\
& a\left(\mathbf{u}^{h}, \mathbf{v}\right)=l(\mathbf{v})
\end{aligned}
$$

which can be solved using classical finite element technology [44], or as in our case, using the Cartesian Grid Finite Element Method, cgFEM [37].

\section{The SPR-C technique. The Nearly equilibrated recovery procedure}

Since the introduction of the plain SPR technique [20] we can find several contributions aimed at improving the quality and the robustness of this technique. In general they couple the stress components in order to be able to add constraints that improve the quality of the recovered field. Wiberg and Abdulwahab [45, 25] proposed to take into account the equilibrium of the recovered field by using a penalty method, Blacker and Belytschko [26] introduced the "Conjoint Polynomial Enhancement" to improve the recovered field along the boundaries. Other techniques looking for equilibrated recovered solutions for upper bounding purposes can be found in [46, 47, 48, 49], but always presenting small lacks of equilibrium even at patch level, thus preventing the strict upper bound property.

More recently, Ródenas and coworkers introduced the so-called SPR-C technique [28], where the "C" stands for "constraint", which was later applied in the XFEM framework by Ródenas et al. [34] and finally adapted to geometry-mesh independent FE formulations [35], such as the cgFEM. We will here show the main features of the SPR-C technique. As in the SPR technique, a patch $\mathcal{P}^{i}$ is defined as the set of elements connected to a vertex node $i$. On each patch, a polynomial expansion for each one of the components of the recovered stress field is considered. It is 
necessary to simultaneously consider all the components of the stress vector to be able to include the required constraint equations. Thus, in the SPR-C technique, the recovered stress field for the $2 \mathrm{D}$ case, for each patch, reads:

$$
\hat{\boldsymbol{\sigma}}_{i}^{*}(\mathbf{x})=\left\{\begin{array}{l}
\hat{\sigma}_{x x}^{*}(\mathbf{x}) \\
\hat{\sigma}_{y y}^{*}(\mathbf{x}) \\
\hat{\sigma}_{x y}^{*}(\mathbf{x})
\end{array}\right\}=\mathbf{P}(\mathbf{x}) \mathbf{a}=\left[\begin{array}{ccc}
\mathbf{p}(\mathbf{x}) & \mathbf{0} & \mathbf{0} \\
\mathbf{0} & \mathbf{p}(\mathbf{x}) & \mathbf{0} \\
\mathbf{0} & \mathbf{0} & \mathbf{p}(\mathbf{x})
\end{array}\right]\left\{\begin{array}{l}
\mathbf{a}_{x x} \\
\mathbf{a}_{y y} \\
\mathbf{a}_{x y}
\end{array}\right\}
$$

The polynomial coefficients a for each component of the stress field are obtained by optimizing the following functional:

$$
\begin{aligned}
\Phi(\mathbf{A}, \boldsymbol{\lambda}):= & \int_{\mathcal{P}^{i}}\left(\mathbf{P}(\mathbf{x}) \mathbf{a}-\boldsymbol{\sigma}^{h}(\mathbf{x})\right)^{2} \mathrm{~d} \Omega+ \\
& \sum_{j}^{\text {niee }} \lambda_{j}^{\mathrm{int}}\left(c^{\mathrm{int}}\left(\mathbf{x}_{j}\right)\right)+\sum_{j}^{\text {nbee }} \lambda_{j}^{\mathrm{bnd}}\left(c^{\mathrm{bnd}}\left(\mathbf{x}_{j}\right)\right)+\sum_{j}^{n c e} \lambda_{j}^{\mathrm{cmp}}\left(c^{\mathrm{cmp}}\left(\mathbf{x}_{j}\right)\right)
\end{aligned}
$$

where the constraints equations $c^{\text {int }}$ (internal equilibrium), $c^{\text {bnd }}$ (boundary equilibrium) and $c^{\mathrm{cmp}}$ (compatibility equation), are imposed via Lagrange multipliers $(\lambda)$. For further details on the SPR-C we address the interested reader to $[28,50]$.

Once the local field, at each patch, is obtained, a continuous field for the whole domain is evaluated using a partition of unity procedure (defined in [26] as Conjoint Polynomial enhancement) that, at any point $\mathbf{x}$, properly weights the stress polynomials $\boldsymbol{\sigma}_{i}^{*}$ obtained from patches corresponding to each one of the vertex nodes $i$ of the element containing $\mathbf{x}$. Thus, the field $\boldsymbol{\sigma}_{\sigma}^{*}$ is constructed as a linear weighting of the contributions of each patch using linear shape functions $N_{i}$ associated with the $n_{v}$ vertex nodes according to the following expression:

$$
\boldsymbol{\sigma}_{\sigma}^{*}(\mathbf{x})=\sum_{i=1}^{n_{v}} N_{i}(\mathbf{x}) \hat{\boldsymbol{\sigma}}_{i}^{*}(\mathbf{x})
$$

Subindex $\sigma$ indicates that the internal equilibrium and boundary equilibrium have been imposed locally at each patch during the recovery process. For further details of the SPR-C recovery process see $[28,30]$. Note that the difference between this approach and the one proposed by Zienkiewicz and Zhu [18] is that in this last case, they only retain the recovered stress value $\hat{\boldsymbol{\sigma}}_{i}^{*}\left(\mathbf{x}_{i}\right)$ at the node, but in our approach, based on the Conjoint Polynomial enhancement, we retain the full polynomial, $\hat{\boldsymbol{\sigma}}_{i}^{*}(\mathbf{x})$, that defines the stress field at each patch.

\subsection{Equilibrium defaults of the recovered field}

When the partition of unity is applied to obtain a continuous recovered stress field (11), we generate lacks of equilibrium in the recovered solution according to the 
following result. For $\mathbf{x}$ in the interior of elements:

$$
\begin{aligned}
\mathbf{L}^{T} \boldsymbol{\sigma}_{\sigma}^{*}(\mathbf{x}) & =\mathbf{L}^{T} \sum_{i=1}^{n_{v}} N_{i}(\mathbf{x}) \hat{\boldsymbol{\sigma}}_{i}^{*}(\mathbf{x}) \\
& =\underbrace{\sum_{i=1}^{n_{v}}\left(\mathbf{L}^{T} N_{i}(\mathbf{x})\right) \hat{\boldsymbol{\sigma}}_{i}^{*}(\mathbf{x})}_{-\mathbf{s}_{\sigma}^{*}}+\sum_{i=1}^{n_{v}} N_{i}(\mathbf{x}) \underbrace{\mathbf{L}^{T} \hat{\boldsymbol{\sigma}}_{i}^{*}(\mathbf{x})}_{\mathbf{L}^{T} \hat{\boldsymbol{\sigma}}_{i}^{*}=-\mathbf{b}} \\
& =-\mathbf{s}_{\sigma}^{*}-\mathbf{b}
\end{aligned}
$$

This expression is a modified version of the internal equilibrium equation where $\mathbf{s}_{\sigma}^{*}$ represents the lack of internal equilibrium. Furthermore, we could also consider a lack of boundary equilibrium of $\boldsymbol{\sigma}_{\sigma}^{*}$ over $\Gamma_{N}$ which can be evaluated as $\mathbf{r}_{\sigma}^{*}=$ $\mathbf{G} \boldsymbol{\sigma}_{\sigma}^{*}-\mathbf{t}_{\Gamma_{N}}$, where $\mathbf{t}_{\Gamma_{N}}$ are the exact tractions over the Neumann boundaries. The recovered field will meet the prescribed traction along straight boundaries if this traction field is in the basis of the recovered field. Note that, in the case of curved boundaries, the polynomial traction field, in general, will not be met by the recovered stress field. It is important to note that, in any case, the lack of equilibrium along the boundaries is negligible in comparison with the lack of internal equilibrium, then $\mathbf{r}_{\sigma}^{*}$ is only formally introduced in equations.

\section{Error estimation. A SPR-based upper bound- ing technique}

In this section we will first introduce a general upper bounding technique of the error in energy norm for general SPR-based recovery procedures. The upper bounding technique presented in this work consists of two parts. The first one is the classical ZZ error estimator, providing of accuracy to the error estimator, and the second part is an explicit-type residual-based error estimator to bound the lack of equilibrium of the recovered stress field, providing the required bounding properties. Then, we will show an alternative procedure based on the residual of the recovered solution to obtain this bound. We will apply the results to the particular case in which the recovered solution is obtained with the SPR-C recovery technique. Finally, we will show a procedure to obtain a numerical value for the constant required for bounding the correction terms when the SPR-C recovery technique is used.

The exact error in the energy norm of the FE solution, which is generally not available for real-life problems, can be written as:

$$
\|\mid \mathbf{e}\| \|_{\Omega}^{2}=\int_{\Omega}\left(\boldsymbol{\sigma}-\boldsymbol{\sigma}^{h}\right)^{T} \mathbf{D}^{-1}\left(\boldsymbol{\sigma}-\boldsymbol{\sigma}^{h}\right) \mathrm{d} \Omega
$$

where $\mathbf{e}=\mathbf{u}-\mathbf{u}^{h}$. Zienkiewicz and Zhu [18] introduced the so-called Zienkiewicz and Zhu (ZZ) error estimator (14) which consists of substituting the unknown field $\boldsymbol{\sigma}$ by the recovered stress field $\boldsymbol{\sigma}^{*}$, evaluated with a recovery procedure, in expression (13).

$$
\mathscr{E}^{2}:=\int_{\Omega}\left(\boldsymbol{\sigma}^{*}-\boldsymbol{\sigma}^{h}\right)^{T} \mathbf{D}^{-1}\left(\boldsymbol{\sigma}^{*}-\boldsymbol{\sigma}^{h}\right) \mathrm{d} \Omega
$$


Thanks to the accuracy of the recovered field evaluated with SPR-type techniques, this asymptotically exact error estimator produces very accurate estimations of the exact error, especially with enhanced versions of the original SPR like the SPR-C technique, where information about the exact solution (equilibrium and compatibility) is considered in the evaluation of $\boldsymbol{\sigma}^{*}$.

However, under this framework, we need a statically admissible stress field in order to obtain an upper bound of the error in the energy norm. This means that this stress field has to be in equilibrium with the body loads and the Neumann tractions. One example of the use of this idea is the Constitutive Relation Error (CRE) introduced by Prof. Ladevèze [11]. In the CRE technique the authors evaluate a stress field $\boldsymbol{\sigma}_{\mathrm{CRE}}$ which is statically admissible. Then, by using an expression similar to (14) they evaluate an upper bound of the error in the energy norm.

The basic SPR-type techniques produce continuous fields $\boldsymbol{\sigma}^{*}$ but they fail to obtain a statically admissible stress field. The SPR-based techniques, in general, introduce a lack of internal equilibrium $\mathbf{s}^{*}$ and boundary equilibrium $\mathbf{r}^{*}$. Díez et. al. [33] introduced an expression to evaluate an upper bound of the error in the energy norm with a correction term to account for the lack of internal equilibrium. A generalization of that expression is introduced in (15), which does not only include the lack of internal equilibrium but also the lack of boundary equilibrium making use of the two correction terms shown in (16):

$$
\begin{aligned}
& \mathscr{E}_{\mathrm{UB}}^{2}:=\mathscr{E}^{2}+\mathscr{E}_{\mathrm{int}}+\mathscr{E}_{\text {bnd }} \\
& \mathscr{E}_{\mathrm{int}}:=-2 \int_{\Omega}\left(\mathbf{s}^{*}\right)^{T} \mathbf{e} \mathrm{d} \Omega \\
& \mathscr{E}_{\text {bnd }}:=-2 \int_{\Gamma_{N}}\left(\mathbf{r}^{*}\right)^{T} \mathbf{e} \mathrm{d} \Gamma
\end{aligned}
$$

These correction terms require the exact displacement solution, e, to be evaluated. In this section we are interested in bounding them in order to obtain guaranteed upper bounds of the error in the energy norm. These correction terms are first bounded with the Cauchy-Schwarz inequality:

$$
\begin{gathered}
\left|\int_{\Omega}\left(\mathbf{s}^{*}\right)^{T} \mathbf{e} \mathrm{d} \Omega\right| \leq\left\|\mathbf{s}^{*}\right\|_{L_{2}(\Omega)}\|\mathbf{e}\|_{L_{2}(\Omega)} \\
\left|\int_{\Gamma_{N}}\left(\mathbf{r}^{*}\right)^{T} \mathbf{e} \mathrm{d} \Gamma\right| \leq\left\|\mathbf{r}^{*}\right\|_{L_{2}\left(\Gamma_{N}\right)}\|\mathbf{e}\|_{L_{2}\left(\Gamma_{N}\right)}
\end{gathered}
$$

As $\mathbf{s}^{*}$ and $\mathbf{r}^{*}$ can be evaluated, the correction terms in (17) will be bounded if we obtain a bound of the $L_{2}$-norm of the error in the displacement field. With the Aubin-Nitsche lemma ([51] p. 136), the $L_{2}$-norm of the error in the displacement field can be bounded with the respective error in the energy norm, then:

$$
\|\mathbf{e}\|_{L_{2}(\Omega)} \leq C_{\Omega} h\|\| \mathbf{e} \|_{\Omega}
$$

Additionally, making use of the trace inequality [5] we can also bound the error evaluated in $L_{2}$-norm over the boundary with the error in energy norm according to the following expression:

$$
\|\mathbf{e}\|_{L_{2}\left(\Gamma_{N}\right)} \leq C_{\Gamma} h^{\frac{1}{2}}\|\| \mathbf{e} \|_{\Omega}
$$


where $C_{\Omega}$ and $C_{\Gamma}$ are constants dependent on the problem but independent on the mesh size, and $h$ is a relative representative size of the mesh (in an $h$-uniform refinement process $h$ is the element size). It is important to remark that standard residual-based error estimators are usually derived using Clément-type inequalities $[5,6]$ thanks to the orthogonality between the FE solution and the error. However, in our case the recovered stress field does not satisfy the Galerkin orthogonality. The inequalities in (18) and (19) were developed for $h$-uniform refinement processes. However they can be extended for constant-pattern $h$-adaptive refinement processes, since for a defined mesh pattern it is possible to find a geometrical mapping that converts the $h$-adapted mesh to an uniform one. The constant-pattern meshes can be found in standard $h$-adaptive refinement processes when the asymptotic range is achieved. The refinement processes start from a mesh of elements of uniform size. During the process, in the pre-asymptotic range, the $h$-adaptive technique will adequately refine the zones which need a smaller element size in order to obtain an equidistribution of the discretization error. Once the equidistribution of the error is achieved, in the asymptotic range, the refinement process essentially consists of a uniform refinement of the $h$-adapted mesh. That can be defined as constant-pattern $h$-adaptive refinement processes. Under this situation, $h$ can be related with the

Number of Degrees of Freedom (NDoF). Therefore we define $h:=\left(\frac{1}{N D o F}\right)^{\frac{1}{d}}$, where $d$ is the dimension of the problem. Then, we can bound the correction terms as follows:

$$
\begin{aligned}
& \mathscr{E}_{\text {int }} \leq 2\left|\int_{\Omega}\left(\mathbf{s}^{*}\right)^{T} \mathbf{e} \mathrm{d} \Omega\right| \leq 2\left\|\mathbf{s}^{*}\right\|_{L_{2}(\Omega)}\|\mathbf{e}\|_{L_{2}(\Omega)} \leq C_{\Omega} h\left\|\mathbf{s}^{*}\right\|_{L_{2}(\Omega)}\|\mathbf{e}\| \|_{\Omega} \\
& \mathscr{E}_{\text {bnd }} \leq 2\left|\int_{\Gamma_{N}}\left(\mathbf{r}^{*}\right)^{T} \mathbf{e} \mathrm{d} \Gamma\right| \leq 2\left\|\mathbf{r}^{*}\right\|_{L_{2}\left(\Gamma_{N}\right)}\|\mathbf{e}\|_{L_{2}\left(\Gamma_{N}\right)} \leq C_{\Gamma} h^{\frac{1}{2}}\left\|\mathbf{r}^{*}\right\|_{L_{2}\left(\Gamma_{N}\right)}\|\| \mathbf{e}\|\|_{\Omega}
\end{aligned}
$$

\subsection{Alternative interpretation of the correction terms}

Let us evaluate the bounds in (17) from the perspective of the residual of the recovered solution whose error is not orthogonal to the exact solution, thus preventing the use of the Galerkin orthogonality. We define the residual associated with the recovered stress field $\boldsymbol{\sigma}^{*}$ as:

$$
R^{*}(\mathbf{v}):=l(\mathbf{v})-\bar{a}\left(\boldsymbol{\sigma}^{*}, \boldsymbol{\sigma}(\mathbf{v})\right) \quad \forall \mathbf{v} \in V
$$

where $\bar{a}(\cdot, \cdot)$ represents the weak form of the elasticity problem in terms of the stress field. This expression can be rewritten as follows:

$$
\begin{aligned}
R^{*}(\mathbf{v})=\sum_{K \in \mathcal{T}}\left(\int_{\Omega^{K}} \mathbf{b}^{T} \mathbf{v} \mathrm{d} \Omega+\int_{\Gamma_{N} \cap \partial \Omega^{K}} \mathbf{t}^{T} \mathbf{v} \mathrm{d} \Gamma\right) \\
\quad-\sum_{K \in \mathcal{T}} \int_{\Omega^{K}}\left(\boldsymbol{\sigma}^{*}\right)^{T} \boldsymbol{\varepsilon}(\mathbf{v}) \mathrm{d} \Omega \quad \forall \mathbf{v} \in V
\end{aligned}
$$


where $K$ is each of the elements of the partition $\mathcal{T}$. Integrating by parts:

$$
\begin{aligned}
R^{*}(\mathbf{v})=\sum_{K \in \mathcal{T}} & \left(\int_{\Omega^{K}} \mathbf{b}^{T} \mathbf{v} \mathrm{d} \Omega+\int_{\partial \Omega^{K} \cap \Gamma_{N}} \mathbf{t}^{T} \mathbf{v} \mathrm{d} \Gamma\right) \\
& +\sum_{K \in \mathcal{T}}\left(\int_{\Omega^{K}}\left(\mathbf{L}^{T} \boldsymbol{\sigma}^{*}\right)^{T} \mathbf{v} \mathrm{d} \Omega-\int_{\partial \Omega^{K}}\left(\mathbf{G} \boldsymbol{\sigma}^{*}\right)^{T} \mathbf{v} \mathrm{d} \Gamma\right) \quad \forall \mathbf{v} \in V
\end{aligned}
$$

grouping terms and using the definition of $-\mathbf{s}^{*}=\mathbf{L}^{T} \boldsymbol{\sigma}^{*}+\mathbf{b}$ in $K$ and, $-\mathbf{r}^{*}=\mathbf{t}-\mathbf{G} \boldsymbol{\sigma}^{*}$ only over $\Gamma_{N}$, we end up with the following expression:

$$
R^{*}(\mathbf{v})=-\sum_{K \in \mathcal{T}}\left(\int_{\Omega^{K}}\left(\mathbf{s}^{*}\right)^{T} \mathbf{v} \mathrm{d} \Omega+\int_{\partial \Omega^{\mathrm{K}} \cap \Gamma_{\mathrm{N}}}\left(\mathbf{r}^{*}\right)^{T} \mathbf{v} \mathrm{d} \Gamma\right) \quad \forall \mathbf{v} \in V
$$

Applying the Cauchy-Schwarz inequality we obtain:

$$
\left|R^{*}(\mathbf{v})\right| \leq\left\|\mathbf{s}^{*}\right\|_{L_{2}(\Omega)}\|\mathbf{v}\|_{L_{2}(\Omega)}+\left\|\mathbf{r}^{*}\right\|_{L_{2}\left(\Gamma_{N}\right)}\|\mathbf{v}\|_{L_{2}\left(\Gamma_{N}\right)} \quad \forall \mathbf{v} \in V
$$

Now, particularizing (25) for $\mathbf{v}=\mathbf{e}$ we obtain we obtain (26) that contains the terms in (17):

$$
\left|R^{*}(\mathbf{e})\right| \leq\left\|\mathbf{s}^{*}\right\|_{L_{2}(\Omega)}\|\mathbf{e}\|_{L_{2}(\Omega)}+\left\|\mathbf{r}^{*}\right\|_{L_{2}\left(\Gamma_{N}\right)}\|\mathbf{e}\|_{L_{2}\left(\Gamma_{N}\right)}
$$

Finally, the upper bounds are obtained applying the inequality defined in (20):

$$
\left|R^{*}(\mathbf{e})\right| \leq C_{\Omega} h\left\|\mathbf{s}^{*}\right\|_{L_{2}(\Omega)}|\|\mathbf{e}\||_{\Omega}+C_{\Gamma} h^{\frac{1}{2}}\left\|\mathbf{r}^{*}\right\|_{L_{2}\left(\Gamma_{N}\right)}\|\mid \mathbf{e}\| \|_{\Omega}
$$

\subsection{Particular case using the SPR-C recovery technique}

Let us now consider the evaluation of the upper bound of the discretization error in the energy norm when the SPR-C technique is used instead of a standard SPRbased procedure: $\boldsymbol{\sigma}^{*}=\boldsymbol{\sigma}_{\sigma}^{*}$. Because of the use of the SPR-C technique, the lack of equilibrium along the boundary is either null or negligible, thus we can neglect the terms related to the lack of equilibrium along the boundaries $\left\|\mathbf{r}_{\sigma}^{*}\right\|_{L_{2}\left(\Gamma_{N}\right)}$. Then, with minimal loss in accuracy:

$$
\left|R^{*}(\mathbf{e})\right| \leq C h\left|\|\mathbf{e} \mid\|_{\Omega}\left\|\mathbf{s}_{\sigma}^{*}\right\|_{L_{2}(\Omega)}\right.
$$

where $C=C_{\Omega}$. Now, we rewrite expression (15) composed by the ZZ error estimator (14) and the correction terms (16) as follows:

$$
\left\||\mathbf{e} \||_{\Omega}^{2} \leq \int_{\Omega}\left(\boldsymbol{\sigma}_{\sigma}^{*}-\boldsymbol{\sigma}^{h}\right)^{T} \mathbf{D}^{-1}\left(\boldsymbol{\sigma}_{\sigma}^{*}-\boldsymbol{\sigma}^{h}\right) \mathrm{d} \Omega+2 \mathrm{R}^{*}(\mathbf{e})=\mathscr{E}^{2}+2 \mathrm{R}^{*}(\mathbf{e})\right.
$$

substituting (28) in (29) we obtain:

$$
\|\mathbf{e}\|\left\|_{\Omega}^{2} \leq \mathscr{E}^{2}+C h\right\| \mid \mathbf{e}\|\|_{\Omega}\left\|\mathbf{s}_{\sigma}^{*}\right\|_{L_{2}(\Omega)}=\mathscr{E}^{2}+\Xi\|\| \mathbf{e} \|_{\Omega}
$$

Expression (30) is a second order degree polynomial in $\left.\||\mathbf{e}|\|\right|_{\Omega}$. The most conservative root provides the upper bound in the energy norm up to the constant $C$, see [33],

$$
\|\mathbf{e}\|_{\Omega} \leq \frac{\Xi+\sqrt{\Xi^{2}+4 \mathscr{E}^{2}}}{2}
$$

Now we have to investigate the convergence rate of each term in (30). Assuming that $p$ is the order of the FE interpolation: 
- $\mathscr{E}$ : this term is considered asymptotically exact because the recovery technique has a higher convergence rate than the FE solution [22]. Thus its convergence rate could be considered the same as that of the error in the energy norm, $p$.

- $\|\mathbf{|} \mid\|_{\Omega}$ : obviously the convergence rate of this term is $p$ for regular solutions.

- $\Xi$ : the convergence rate of this term is not totally straightforward. In our case, it will depend on the convergence rate of $\left\|\mathbf{s}_{\sigma}^{*}\right\|_{L_{2}(\Omega)}$. Assuming that the recovered field has a convergence rate $p+q, q>0$, higher than that for the FE solution, $p$, the convergence rate of $\left\|\mathbf{s}_{\sigma}^{*}\right\|_{L_{2}(\Omega)}$ would be $p+q-1$. Then, under this situation, the convergence rate of $\Xi$ could be considered as $p+q$.

Although we cannot guarantee superconvergence $(q=1)$ we know that the recovered field converges faster than the FE solution, $q>0$ [52]. This means that the correction terms tend to vanish along the refinement process. Therefore, the plain ZZ error estimator with the SPR-C technique will provide asymptotically guaranteed upper bounds of the error in energy norm.

Finally, we can conclude that provided the recovered field converges faster than the FE solution, we obtain a stable upper bound. With regards to the constant $C$, some authors take the value $C=1$ for convergence purposes [5]. However, for error estimation purposes, this approach could be inaccurate in some cases and in others it would provide under estimations of the true error. In the following section we will propose a methodology to numerically compute this constant for each problem.

\subsection{Numerical evaluation of the constant $C_{\Omega}$}

Expression (31) is an upper bound of the error in the energy norm, but requires the evaluation of a constant $C_{\Omega}$ which is specific for each problem and also for each discretization type. This constant relates the $L_{2}$-norm of the error in displacements with the respective error in the energy norm, as shown in (18).

Prof. Stein's group at Leibniz University is actively working in explicit residualtype error estimators for the elasticity problem $[5,53]$. These error estimators also require the evaluation of a constant with similar characteristics to the constant used in (18), but arising from the Korn inequality. Recently, Prof. Stein and coworkers presented a value for the constant used in their explicit residual-type error estimator $[8,9]$, valid for linear triangular elements.

In this work we propose a methodology to numerically estimate the value of the constant $C_{\Omega}$ to be used in (31) for each problem. We assume that we are under an $h$-refinement process which is required to evaluate the solution with the level of accuracy defined by the user. Therefore, a set of $h$-adapted meshes will be available. Let $H$ and $h$ be the representative sizes of two meshes such that $H<<h$ being $\mathbf{u}^{H}$ the FE solution of the finer mesh. We can consider that $\mathbf{u}^{H}$ is a good approximation to $\mathbf{u}$ in comparison with $\mathbf{u}^{h}$. Considering the Richardson extrapolation the following 
relations will hold:

$$
\begin{aligned}
\left\|\mathbf{u}-\mathbf{u}^{h}\right\|_{L_{2}(\Omega)}^{2} & \approx \frac{\left\|\mathbf{u}^{H}-\mathbf{u}^{h}\right\|_{L_{2}(\Omega)}^{2}}{1+\left(\frac{H}{h}\right)^{2 p+2}} \\
\left\|\mathbf{u}-\mathbf{u}^{h}\right\|_{\Omega}^{2} & \approx \frac{\left.\left\|\mathbf{u}^{H}-\mathbf{u}^{h}\right\|\right|_{\Omega} ^{2}}{1+\left(\frac{H}{h}\right)^{2 p}}
\end{aligned}
$$

where $p$ is the degree of the FE solution. In order to keep the information of the solution in the finer mesh, the integration will be performed in the finer mesh. Under these assumptions, it easily follows the evaluation of the numerical approximation $C_{\Omega}^{*}$ to $C_{\Omega}$ for the mesh $H$.

$$
C_{\Omega} \approx C_{\Omega}^{*}=\sqrt{\frac{\left\|\mathbf{u}^{H}-\mathbf{u}^{h}\right\|_{L_{2}(\Omega)}^{2}\left(1+\left(\frac{H}{h}\right)^{2 p}\right)}{h^{2}\left\|\mathbf{u}^{H}-\mathbf{u}^{h}\right\|_{\Omega}^{2}\left(1+\left(\frac{H}{h}\right)^{2 p+2}\right)}}
$$

Then the constant $C$ will be also approximated by $C^{*}=C_{\Omega}^{*}$. For other recovery processes, where the boundary equilibrium is not fulfilled, such as in the case of the plain SPR, this cannot be assumed. The numerical evaluation of $C$ can be performed during the refinement process for each mesh $n$, using information from previous meshes. For the first one, since no previous information is available, it will be considered that $C=1$. For the second mesh, the constant will be evaluated comparing the solutions of the first $\mathbf{u}^{1}$ and second $\mathbf{u}^{2}$ meshes. Therefore, $\mathbf{u}^{H}=\mathbf{u}^{2}$ and $\mathbf{u}^{h}=\mathbf{u}^{1}$. From the third mesh, $n>2$, we take, in general, $\mathbf{u}^{H}=\mathbf{u}^{n}$ and $\mathbf{u}^{h}=\mathbf{u}^{n-2}$. Note that this process will only be required for the initial meshes of the $h$-adaptive process until a stable value of the constant is achieved. The use of the cgFEM code is important in this point due to the fact that as it is based on the use of nested meshes, the projection process here described required to evaluate the constant is considerably simplified.

Finally the upper bound of the error in the energy norm reads:

$$
\|\mathbf{e}\|_{\Omega}^{2} \lesssim \hat{\mathscr{E}}_{\mathrm{UB}}:=\frac{\Xi^{*}+\sqrt{\left(\Xi^{*}\right)^{2}+4 \mathscr{E}^{2}}}{2}
$$

where:

$$
\Xi^{*}=2 C^{*} h\left\|\mathbf{s}_{\sigma}^{*}\right\|_{L_{2}(\Omega)}^{2}
$$

\section{$5 \quad$ Numerical Results}

In this Section we will show some numerical evidences of the behavior of this new error bounding technique. We will compare it with the Zienkiewicz and Zhu error estimator (14) based on the use of the SPR-C technique, which presents a high accuracy but does not have bounding properties. We have used two benchmark problems with analytical solution and a problem where only overkilled solution will be available in order to study the behavior of $Q_{1}$ (bilinear) and $Q_{2}$ (biquadratic) elements. 
In order to evaluate the accuracy of the estimates we use the global effectivity index as indicator for the different techniques according to the following expression:

$$
\begin{aligned}
\theta & =\frac{\mathscr{E}}{\|\mid \mathbf{e}\|_{\Omega}} \\
\hat{\theta}_{\mathrm{UB}} & =\frac{\hat{\mathscr{E}}_{\mathrm{UB}}}{\|\mathbf{e}\|_{\Omega}} \\
\theta_{\mathrm{UB}} & =\frac{\mathscr{E}_{\mathrm{UB}}}{\|\mathbf{e}\|_{\Omega}}
\end{aligned}
$$

As pointed out above, the SPR-C technique does not have bounding properties, hence in some situations it will underestimate $(\theta<1)$ the exact error. Despite of that, it usually overestimates of the exact error in the energy norm because of the equilibrating techniques used during the recovery process. The results of the new proposed technique are represented by $\hat{\theta}_{\mathrm{UB}}$. $\theta_{\mathrm{UB}}$ represents the effectivity index provided by (15), in which the exact displacement solution has been used. The value of $\theta_{\mathrm{UB}}$ is used for comparison purposes.

There are two main objectives in the following numerical tests. The first one is to check the quality of the estimate provided by the proposed technique and also its stability and convergence along the refinement process. The second objective is to check the quality in the evaluation of the constant that characterizes equation (18), the key ingredient of the proposed technique for its use in practical applications.

\subsection{Problem 1: $3^{\text {rd }}$ order polynomial displacements in a $2 \times 2$ square}

The first benchmark problem considers an infinite domain problem where we have extracted a $2 \times 2$ square domain, centered at the origin of coordinates. The exact displacement solution is a $3^{\text {rd }}$ order polynomial, with linear body loads over the domain. A model of the problem and the analytical solution considering plane strain conditions is shown in Figure 1. We have imposed the corresponding Neumann boundary conditions.

Figure 2 shows an example of the $h$-adaptive refinement process for linear elements. We observe that the refinement process tends to generate an $h$-uniform refinement form the previous $h$-adapted mesh.

Tables 1 and 2 present the results for this academic problem for different number of degrees of freedom (DoF). In both tables the second, third and fourth columns are the effectivity indices for the techniques as indicated in (36). The last column corresponds to the constant $C_{\Omega}^{*}$ evaluated using (33). An analytical value of the constant is also evaluated, for comparison purposes, from (18) since the analytical solution is known. In order to have a better understanding of the results, they are plotted in Figures 3 and 4, where we can easily check the stability of the constant. In these tables we observe both, the high accuracy of the SPR-C technique and the ability of the proposed techniques to provide accurate upper bound of the error in energy norm. Table 2 shows an example where the plain SPR-C technique was unable to provide upper bounds of the error for most meshes whereas the proposed 


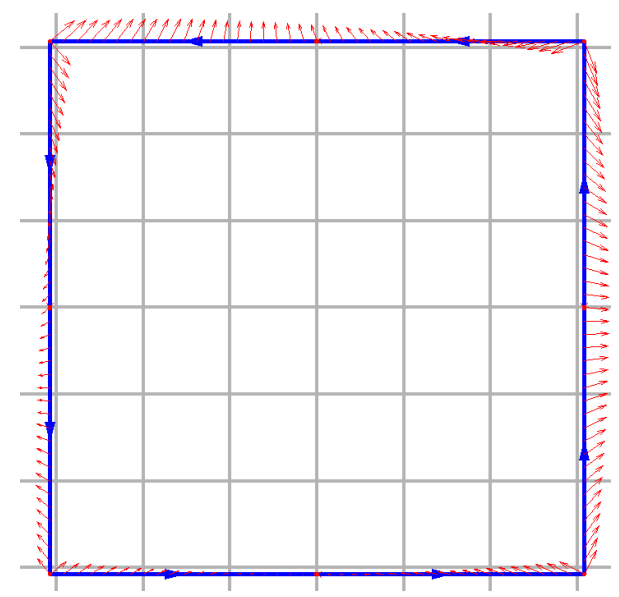

$$
\begin{aligned}
& u_{x}(\mathbf{x})=x+x^{2}-2 x y+x^{3}-3 x y^{2}+x^{2} y \\
& u_{y}(\mathbf{x})=-y-2 x y+y^{2}-3 x^{2} y+y^{3}-x y^{2} \\
& \sigma_{x x}(\mathbf{x})=\frac{E}{1+\nu}\left(1+2 x-2 y+3 x^{2}-3 y^{2}+2 x y\right) \\
& \sigma_{y y}(\mathbf{x})=\frac{-E}{1+\nu}\left(1+2 x-2 y+3 x^{2}-3 y^{2}+2 x y\right) \\
& \sigma_{x y}(\mathbf{x})=\frac{E}{1+\nu}\left(-x-y+\frac{x^{2}}{2}-\frac{y^{2}}{2}-6 x y\right) \\
& b_{x}(\mathbf{x})=\frac{-E}{1+\nu}(1+y) \quad b_{y}(\mathbf{x})=\frac{-E}{1+\nu}(1-x) \\
& E=1000 \quad \nu=0.3
\end{aligned}
$$

Figure 1: Problem 1. Problem model and analytical solution.
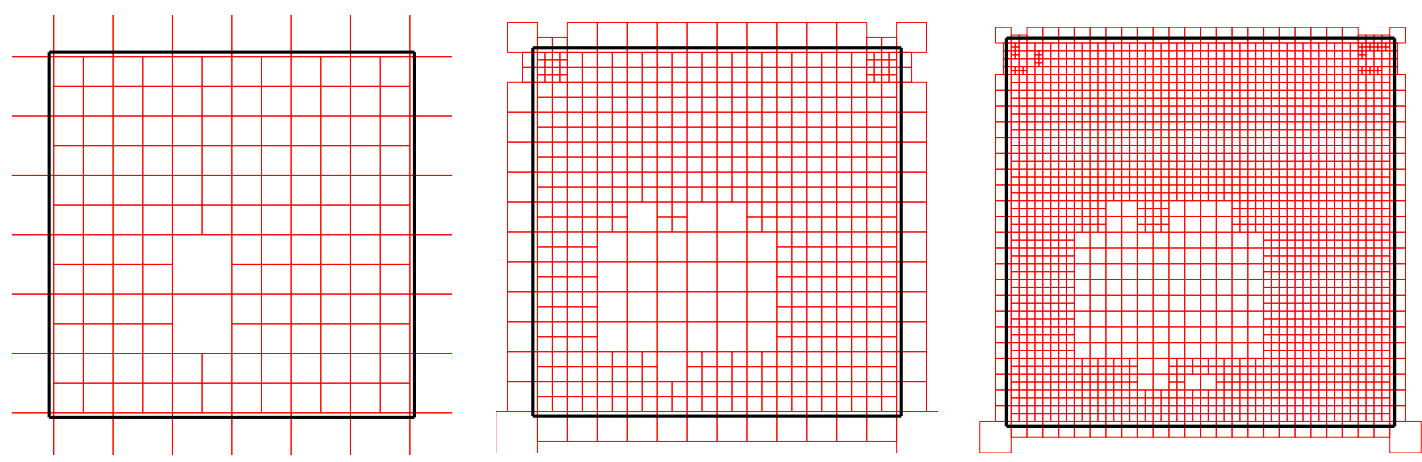

Figure 2: Problem 1. Example of the $2^{\text {nd }}, 3^{\text {rd }}$ and $4^{\text {th }}$ meshes of the $h$-adaptive refinement process for $Q_{1}$ elements.

technique successfully did. Additionally we also observe that the value of the constant stabilizes in a few iterations. In practice, once this constant is stable we do not need to evaluate it again. However, for this work we have evaluated it for all iterations.

Table 1: Problem 1: $Q_{1}$ uniform refinement. Values of the global effectivity index and values of $C_{\Omega}^{*}$. $C_{\Omega}=0.0262472$.

\begin{tabular}{rcccc}
\hline DoF & $\theta$ & $\hat{\theta}_{\mathrm{UB}}$ & $\theta_{\mathrm{UB}}$ & $C_{\Omega}^{*}$ \\
\hline 162 & 1.0146049 & 6.7673407 & 1.0189364 & 1.0000000 \\
450 & 1.0102534 & 1.0991150 & 1.0114409 & 0.0316774 \\
1,458 & 1.0045389 & 1.0793213 & 1.0046972 & 0.0365380 \\
5,202 & 1.0027961 & 1.0504319 & 1.0023176 & 0.0306169 \\
20,402 & 1.0015622 & 1.0312226 & 1.0017645 & 0.0275589 \\
79,202 & 1.0006664 & 1.0203929 & 1.0005893 & 0.0258256 \\
312,050 & 1.0006870 & 1.0176077 & 1.0007558 & 0.0256162 \\
\hline
\end{tabular}

Table 3 and Figure 5 show the stability and the accuracy of the results for $Q_{2}$ elements. Note that, the proposed technique just requires to run a smoothing process, as in the case of the SPR-C technique. Once $C_{\Omega}^{*}$ has taken stable values, 



Figure 3: Problem 1. $Q_{1}$ uniform refinement. Global effectivity index and evolution of $C_{\Omega}^{*}$. $C_{\Omega}=0.0262472$ is represented by a black horizontal line.

Table 2: Problem 1: $Q_{1}$ adaptive refinement. Values of the global effectivity index and values of $C_{\Omega}^{*}$. $C_{\Omega}=0.0468082$.

\begin{tabular}{rcccc}
\hline DoF & $\theta$ & $\hat{\theta}_{\mathrm{UB}}$ & $\theta_{\mathrm{UB}}$ & $C_{\Omega}^{*}$ \\
\hline 162 & 1.0146049 & 6.7673407 & 1.0189364 & 1.0000000 \\
402 & 0.9895298 & 1.0908281 & 1.0098239 & 0.0274138 \\
1,306 & 0.9973276 & 1.1084523 & 1.0059776 & 0.0347587 \\
4,572 & 1.0011230 & 1.1569191 & 1.0046648 & 0.0548123 \\
17,028 & 1.0031465 & 1.1271955 & 1.0069948 & 0.0559684 \\
66,898 & 0.9992628 & 1.0705486 & 1.0017352 & 0.0557396 \\
263,818 & 0.9999673 & 1.0521117 & 1.0011189 & 0.0512197 \\
\hline
\end{tabular}

the additional computational cost to evaluate the bound of the correction terms is negligible as it only requires the evaluation of some integrals not requiring to solve any additional system of equations.

Table 3: Problem 1: $Q_{2}$ adaptive refinement. Values of the global effectivity index and values of $C_{\Omega}^{*}$. $C_{\Omega}=0.0324041$.

\begin{tabular}{rcccc}
\hline DoF & $\theta$ & $\hat{\theta}_{\mathrm{UB}}$ & $\theta_{\mathrm{UB}}$ & $C_{\Omega}^{*}$ \\
\hline 450 & 1.0649543 & 4.1466626 & 1.0573583 & 1.00000000 \\
1,146 & 1.0341480 & 1.0844627 & 1.0382445 & 0.0298479 \\
2,274 & 1.0877080 & 1.1273511 & 1.0813172 & 0.0299108 \\
3,994 & 1.0563165 & 1.1196416 & 1.0520827 & 0.0424869 \\
14,842 & 1.0672356 & 1.1079471 & 1.0607144 & 0.0329398 \\
57,274 & 1.0680699 & 1.1024754 & 1.0625109 & 0.0360767 \\
227,386 & 1.0688648 & 1.1039230 & 1.0615595 & 0.0329120 \\
\hline
\end{tabular}

Figure 6 shows a convergence analysis of the FE solution evaluated with the upper bound proposed in this contribution and compares it with the exact error. The same figure also represents the convergence rate of the term $\Xi$ and of the FE solution. In section 4 we assumed that as a consequence of the use of an SPR-type 

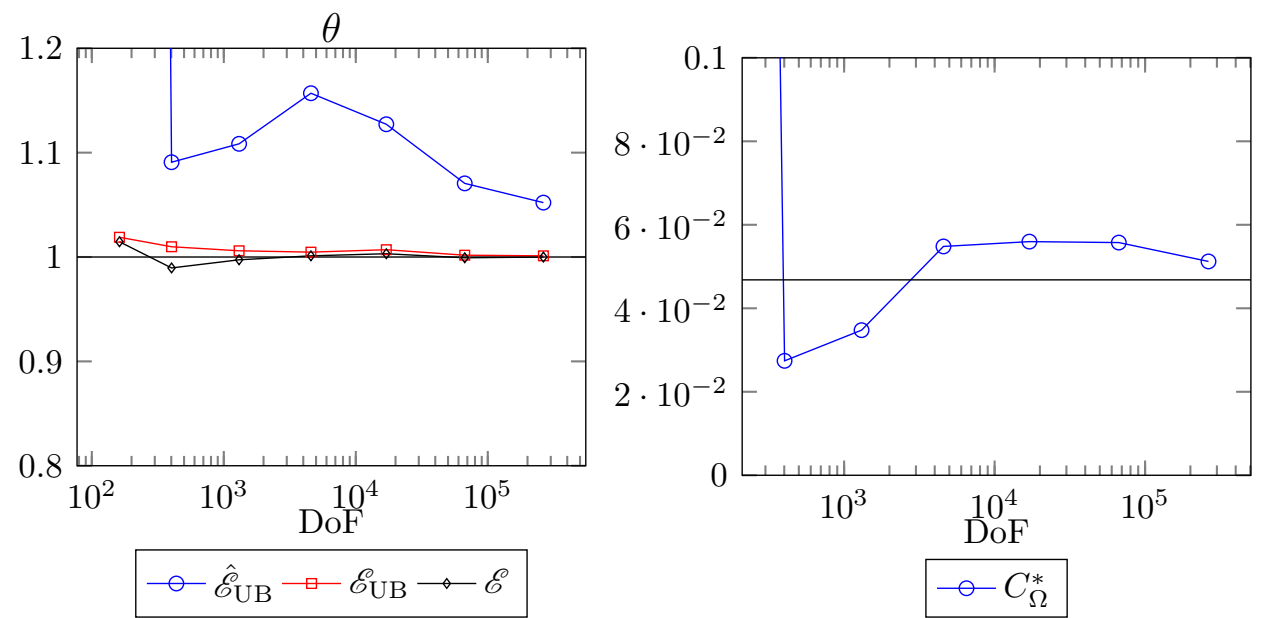

Figure 4: Problem 1. $Q_{1}$ adaptive refinement. Global effectivity index and evolution of $C_{\Omega}^{*}$. $C_{\Omega}=0.0468082$ is represented by a black horizontal line.
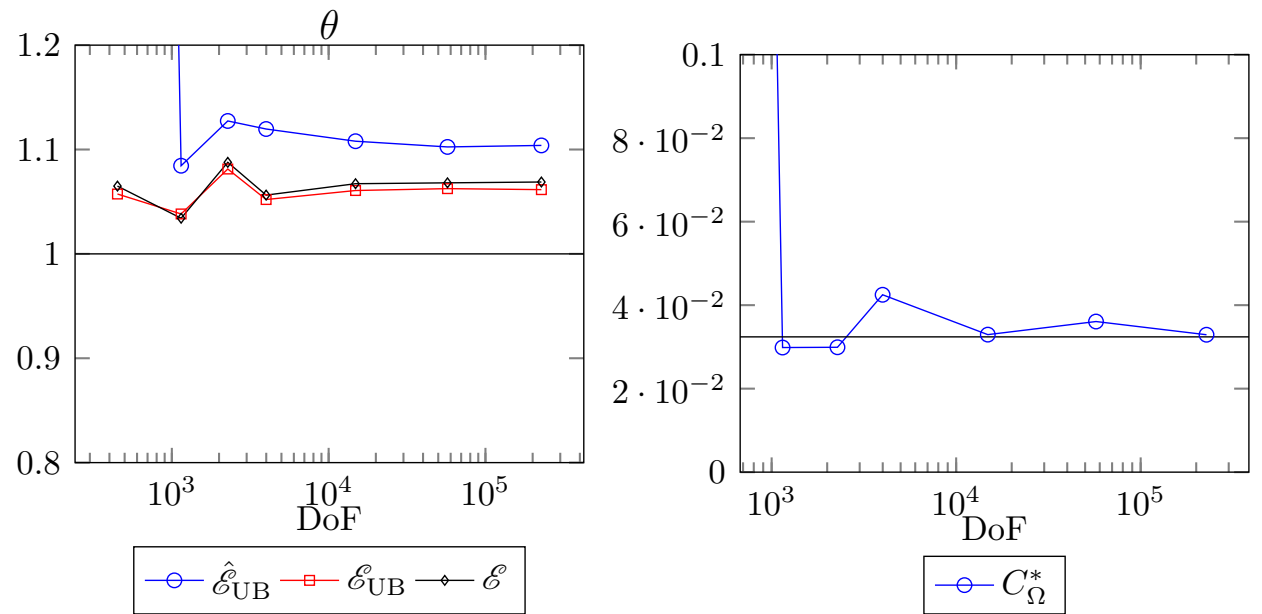

Figure 5: Problem 1. $Q_{2}$ adaptive refinement. Global effectivity index and evolution of $C_{\Omega}^{*}$. $C_{\Omega}=0.0324041$ is represented by a black horizontal line.

technique, the convergence rate of $\Xi$ is $p+q, q>0$, where $p$ is the order of the FE interpolation that would lead to convergence rates of the discretization error in energy norm of values 0.5 for $Q_{1}$ and 1 for $Q_{2}$ in terms of the number of degrees of freedom. In the figure we observe that this assumption holds, ensuring the stability of the method for this problem. 

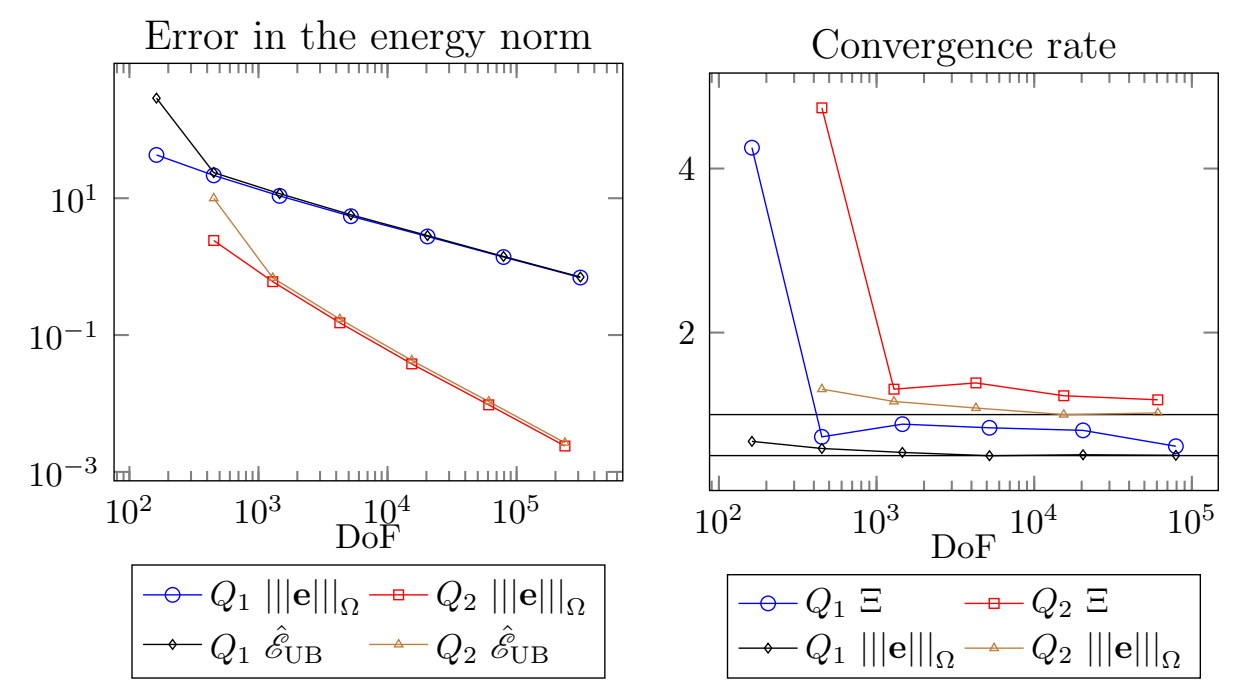

Figure 6: Problem 1. Uniform refinement. Convergence rate of the FE solution and the term $\Xi$. 


\subsection{Problem 2. Thick wall cylinder under internal pressure}

The geometrical model for this problem is represented in Figure 7. Due to symmetry, only $1 / 4$ of the section is modeled. The internal and external surfaces are of radius $a$ and $b$. Young's modulus is $E=1000$, Poisson's ratio is $\nu=0.3, a=5, b=20$ and the internal pressure $P=1$.

The exact solution for the radial displacement assuming plane strain conditions is given by

$$
u_{r}(r)=\frac{P(1+\nu)}{E\left(c^{2}-1\right)}\left(r(1-2 \nu)+\frac{b^{2}}{r}\right)
$$

where $c=b / a, r=\sqrt{x^{2}+y^{2}}$ and $\phi=\arctan (y / x)$. Stresses in cylindrical coordinates are

$$
\sigma_{r}(r)=\frac{P}{c^{2}-1}\left(1-\frac{b^{2}}{r^{2}}\right) \quad \sigma_{\phi}(r)=\frac{P}{c^{2}-1}\left(1+\frac{b^{2}}{r^{2}}\right) \quad \sigma_{z}(r, \phi)=2 \nu \frac{P}{c^{2}-1}
$$

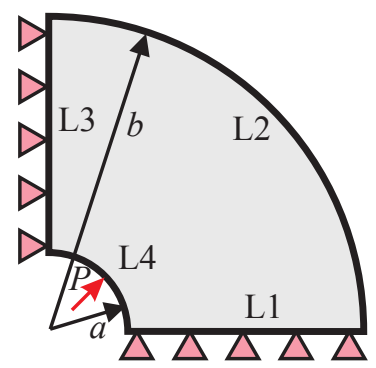

Figure 7: Problem 2. Thick-wall cylinder under internal pressure.

Figure 8 shows an example of the $h$-adaptive refinement process for linear elements. We also observe in this case that the refinement process tends to produce a constant-pattern $h$-refinement.


Figure 8: Problem 2. Example of the $2^{\text {nd }}, 3^{\text {rd }}$ and $4^{\text {th }}$ meshes of the $h$-adaptive refinement process for $Q_{1}$ elements.

Tables 4 and 5 present the results for $Q_{1}$ elements with uniform and $h$-adaptive refinements. The tables show the value of the effectivity indices shown in (36). The values of $C_{\Omega}^{*}$ and $C_{\Omega}$ have also been presented. As in the previous example, the bounding techniques provide upper bounds of the error in energy norm. Note 
that the proposed technique is providing very accurate results once the constant has been stabilized. Note also that the effectivity index $\hat{\theta}_{\mathrm{UB}}$ asymptotically tends to 1 form above due to the higher convergence rate of $\Xi$ as explained before. For further clarity we have also plotted the results of the tables in graphs. Figures 9 and 10 show that the value of $C_{\Omega}^{*}$ tends to stabilize very fast.

Table 4: Problem 2: $Q_{1}$ uniform refinement. Values of the global effectivity index and values of $C_{\Omega}^{*}$. $C_{\Omega}=0.4115025$.

\begin{tabular}{rcccc}
\hline DoF & $\theta$ & $\hat{\theta}_{\mathrm{UB}}$ & $\theta_{\mathrm{UB}}$ & $C_{\Omega}^{*}$ \\
\hline 136 & 1.0674127 & 2.3178582 & 1.1000722 & 1.0000000 \\
448 & 0.9967545 & 1.2063663 & 1.0026439 & 0.3322428 \\
1,488 & 0.9989255 & 1.1737539 & 1.0053102 & 0.4023211 \\
5,542 & 1.0015690 & 1.1311651 & 1.0045074 & 0.4345484 \\
21,016 & 1.0013818 & 1.0855412 & 1.0026123 & 0.4133005 \\
81,752 & 1.0008699 & 1.0593801 & 1.0012975 & 0.4086471 \\
\hline
\end{tabular}
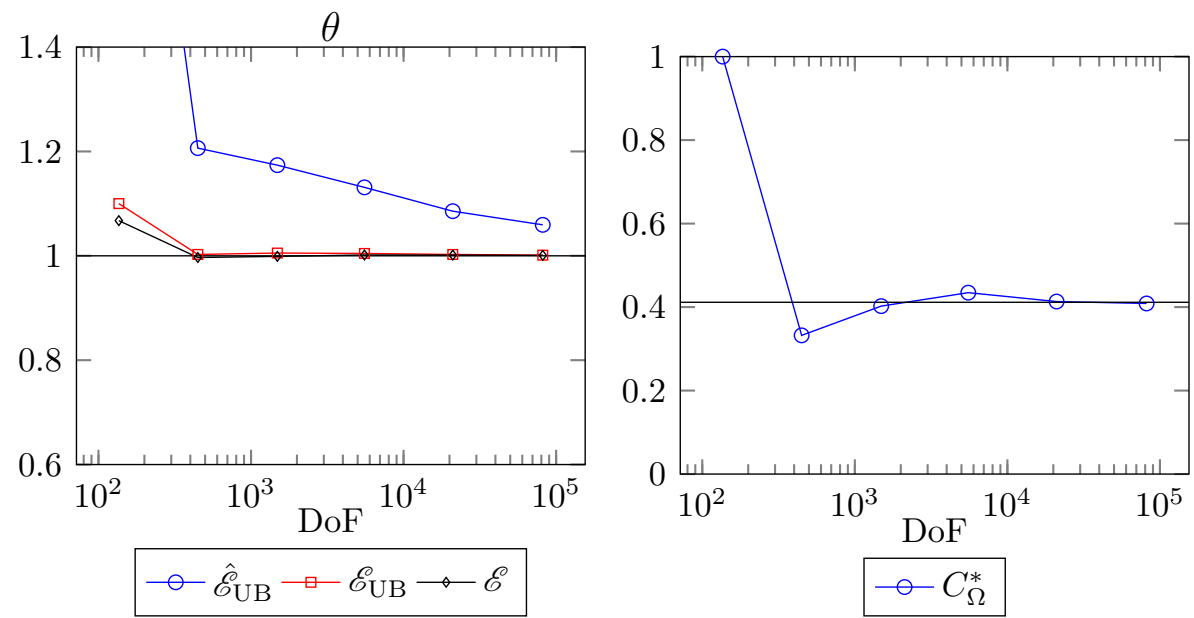

Figure 9: Problem 2. $Q_{1}$ uniform refinement. Global effectivity index and evolution of $C_{\Omega}^{*}$. $C_{\Omega}=0.4115025$ is represented by a black horizontal line.

Table 5: Problem 2: $Q_{1}$ adaptive refinement. Values of the global effectivity index and values of $C_{\Omega}^{*}$. $C_{\Omega}=0.3261038$.

\begin{tabular}{rcccc}
\hline DoF & $\theta$ & $\hat{\theta}_{\mathrm{UB}}$ & $\theta_{\mathrm{UB}}$ & $C_{\Omega}^{*}$ \\
\hline 144 & 1.0505044 & 2.2643341 & 1.0879356 & 1.0000000 \\
240 & 0.9849224 & 1.2969408 & 1.0069175 & 0.3305752 \\
728 & 0.9924221 & 1.3102539 & 1.0062315 & 0.4077924 \\
2,506 & 0.9966148 & 1.2115761 & 1.0060951 & 0.3510156 \\
9,268 & 0.9946859 & 1.1931996 & 1.0034969 & 0.3730436 \\
35,386 & 0.9969832 & 1.1615246 & 1.0022641 & 0.3569402 \\
136,892 & 0.9984190 & 1.1236628 & 1.0020865 & 0.3492249 \\
\hline
\end{tabular}



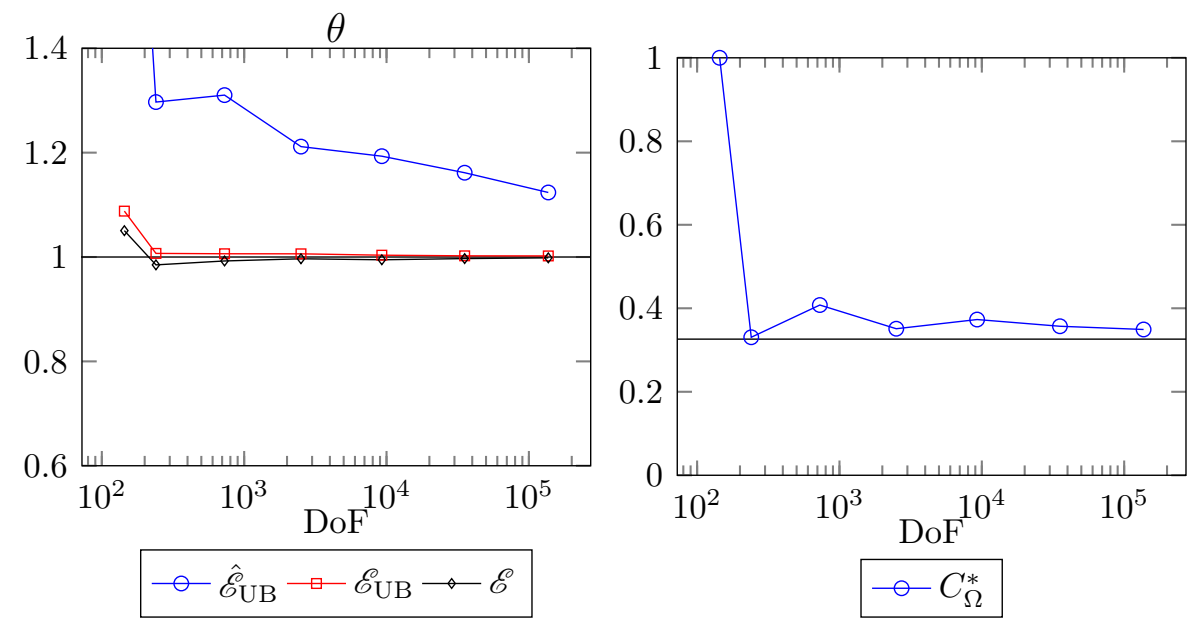

Figure 10: Problem 2. $Q_{1}$ adaptive refinement. Global effectivity index and evolution of $C_{\Omega}^{*}$. $C_{\Omega}=0.3261038$ is represented by a black horizontal line.

Additionally we also present the results for $Q_{2}$ elements under an $h$-adaptive refinement process. We observe that the bounding properties of the proposed technique hold. The method proposed to evaluate the constant $C_{\Omega}^{*}$ provides results very close to the exact value $C_{\Omega}$.

Table 6: Problem 2: $Q_{2}$ adaptive refinement. Values of the global effectivity index and values of $C_{\Omega}^{*}$. $C_{\Omega}=0.3550251$.

\begin{tabular}{rcccc}
\hline DoF & $\theta$ & $\hat{\theta}_{\text {UB }}$ & $\theta_{\text {UB }}$ & $C_{\Omega}^{*}$ \\
\hline 392 & 1.7245828 & 4.2013712 & 1.7481814 & 1.0000000 \\
632 & 1.4077187 & 4.0277798 & 1.3449469 & 0.7163547 \\
1,122 & 1.2116244 & 3.1025755 & 1.1543018 & 0.7316535 \\
2,436 & 1.0981045 & 1.6949636 & 1.0753485 & 0.3645869 \\
4,912 & 1.0692987 & 1.5418848 & 1.0638670 & 0.4168756 \\
7,760 & 1.0740657 & 1.2972085 & 1.0570493 & 0.3096464 \\
12,720 & 1.0618721 & 1.3549504 & 1.0522574 & 0.4470141 \\
28,676 & 1.0630779 & 1.2030182 & 1.0540084 & 0.3445621 \\
49,208 & 1.0656931 & 1.1816800 & 1.0581345 & 0.3352375 \\
109,754 & 1.0578616 & 1.1765046 & 1.0534827 & 0.3541659 \\
\hline
\end{tabular}



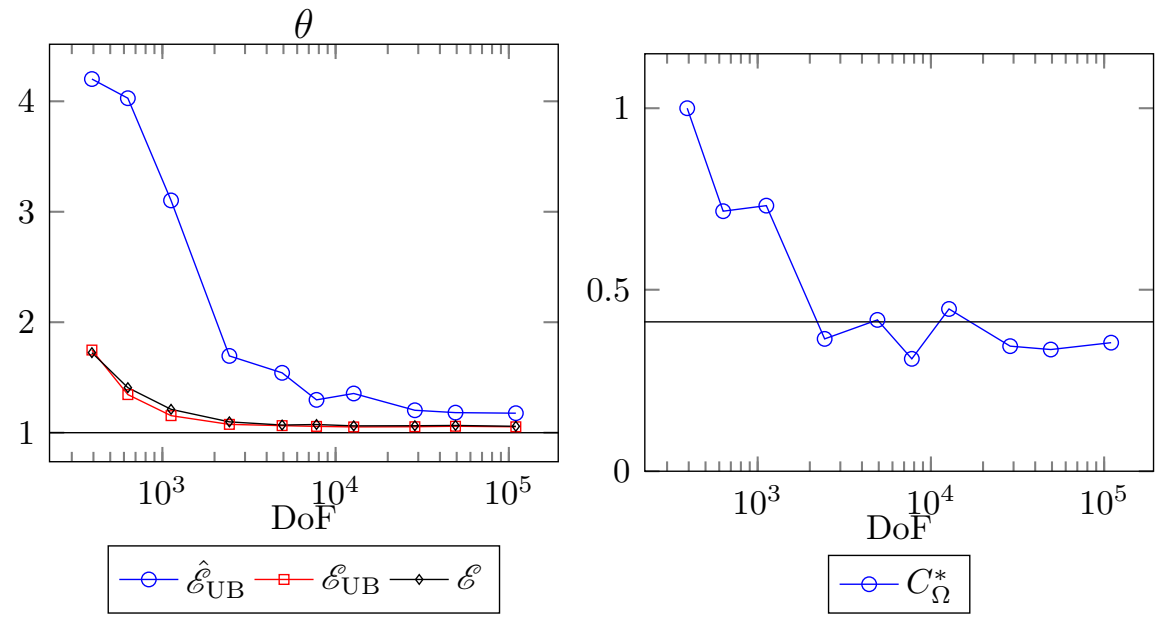

Figure 11: Problem 2. $Q_{2}$ adaptive refinement. Global effectivity index and evolution of $C_{\Omega}^{*}$. $C_{\Omega}=0.4115025$ is represented by a black horizontal line. 


\subsection{Problem 3. Gravity dam}

In this case, we have chosen a more complex geometry in order to check the behavior of the proposed technique in a more realistic environment. For this problem we do not have an analytical solution, thus we will take an overkill solution of 1.933.038 degrees of freedom with biquadractic interpolation as the exact solution in order to evaluate the global effectivity index.

This problem represents a cross section of a gravity dam. The dam is under hydrostatic pressure on the left side. The normal displacement on the three inferior boundaries is constrained, as represented in Figure 12. The material of the dam is concrete with density $\rho_{\text {concrete }}=2300 \frac{\mathrm{kg}}{\mathrm{m}^{3}}$. The density of the water is considered as $\rho_{\text {water }}=1000 \frac{\mathrm{kg}}{\mathrm{m}^{3}}$. The Young's modulus of the concrete is taken as $27.5 \cdot 10^{9} \mathrm{~Pa}$ and the Poisson's ratio $\nu=0.3$. The volume load due to gravity $g=9.81 \frac{\mathrm{m}}{\mathrm{s}^{2}}$ is also considered. We assume plane strain behavior.

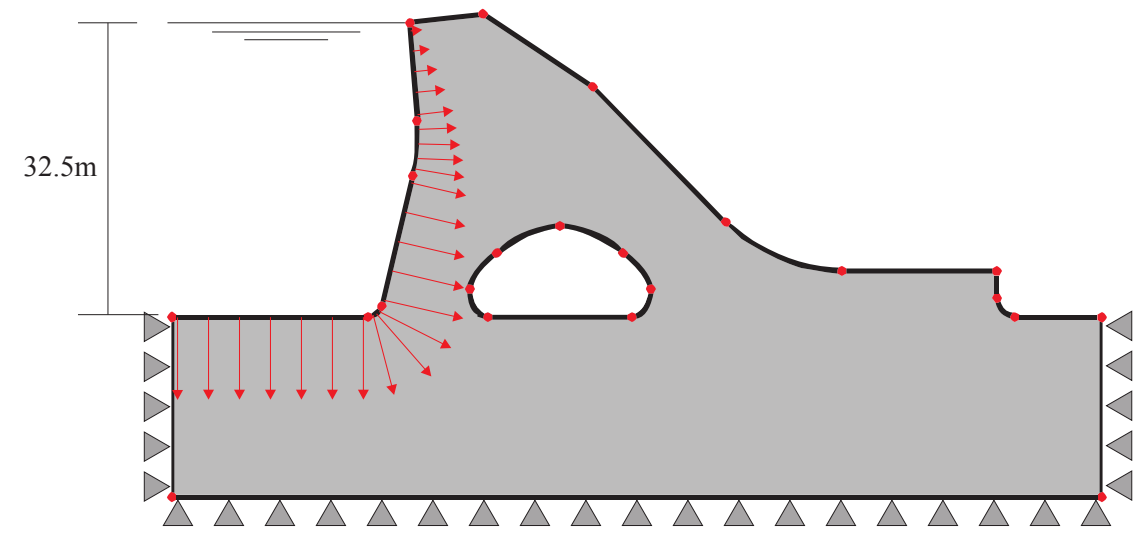

Figure 12: Problem 3. Gravity dam: Model of the problem, loads and constrains.

Figure 13 shows an example of the $h$-adaptive refinement process for linear elements. We observe again a behavior similar to the behavior shown in the previous examples. The $h$-adaptive refinement algorithm tends to produce the constantpattern $h$-refinement process.
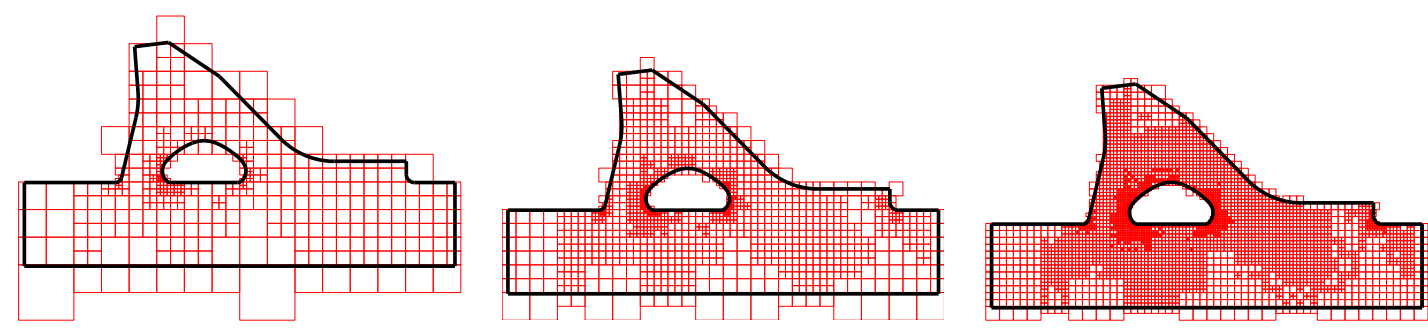

Figure 13: Problem 2. Example of the $2^{\text {nd }}, 3^{\text {rd }}$ and $4^{\text {th }}$ meshes of the $h$-adaptive refinement process for $Q_{1}$ elements.

Table 7 presents the results obtained with the SPR-C technique and the proposed bounding technique. In the first place we observe the value of the estimated error in the energy norm. For the SPR-C technique we obtain a good behavior however for the proposed technique, the first value is extremely high due to the initialization to 
1 of the constant $C_{\Omega}^{*}$. In the second mesh, once the constant has been numerically evaluated, the error measure rapidly decreases to more reasonable values. As indicated above, we have used an overkill solution $\left(Q_{2}\right.$ uniform mesh with 1,933,038 degrees of freedom) to be able to evaluate the global effectivity indices. Note that ' indicates that the effectivity index is evaluated with respect to the overkill solution. The results indicate that the SPR-C technique, provides accurate results, but fails to provide upper bounds of the error in the energy norm. However, the proposed technique, once the constant $C_{\Omega}^{*}$ takes stable values, is providing upper bounds that are smoothly converging to 1 , confirming again the asymptotic exactness of the method. The last column shows the results related with the evaluation of $C_{\Omega}^{*}$, that are stable and smoothly convergent to a constant value.

Table 7: Problem 3: $Q_{1}$ adaptive refinement. Values of relative error in the energy norm $\mathscr{E}$ and $\hat{\mathscr{E}}_{\mathrm{UB}}$, the global effectivity index and values of $C_{\Omega}^{*}$.

\begin{tabular}{rrrrrr}
\hline DoF & $\mathscr{E}_{\mathrm{SPR}-\mathrm{C}}$ & \multicolumn{1}{c}{$\mathscr{E}_{\mathrm{FU}} \mathrm{B}_{\mathrm{SPR}-\mathrm{C}}$} & \multicolumn{1}{c}{$\theta_{\mathrm{SPR}-\mathrm{C}}^{\prime}$} & \multicolumn{1}{c}{$\theta_{\mathrm{FUB}}^{\prime}{ }_{\mathrm{SPR}-\mathrm{C}}$} & \multicolumn{1}{c}{$C_{\Omega}^{*}$} \\
\hline 396 & 0.2121758 & $2,552.6188000$ & 1.2621835 & $15,184.9271000$ & 1.0000000 \\
1,114 & 0.0881817 & 0.2999209 & 1.0412340 & 3.5414139 & 0.0002799 \\
3,362 & 0.0445110 & 0.1316917 & 1.0125846 & 2.9958632 & 0.0003394 \\
11,070 & 0.0225501 & 0.0453444 & 0.9918757 & 1.9944961 & 0.0003154 \\
39,176 & 0.0115666 & 0.0194594 & 0.9943358 & 1.6728504 & 0.0002871 \\
138,942 & 0.0060140 & 0.0089267 & 0.9964700 & 1.4790768 & 0.0002635 \\
496,328 & 0.0031396 & 0.0043132 & 0.9971845 & 1.3699404 & 0.0002481 \\
\hline
\end{tabular}

Table 8 shows the results of the same analysis for $Q_{2}$ elements. We observe the same problem for the first mesh related with the initialization of the constant $C_{\Omega}^{*}$, hence the importance of its numerical evaluation. As shown in the table, both techniques provide upper bounds of the error in energy norm in this case. Regarding the value of the constant $C_{\Omega}^{*}$, in this case it is also stable and convergent along the refinement process.

Table 8: Problem 3: $Q_{2}$ adaptive refinement. Values of relative error in the energy norm $\mathscr{E}$ and $\hat{\mathscr{E}}_{\mathrm{UB}}$, the global effectivity index and values of $C_{\Omega}^{*}$.

\begin{tabular}{rcrrrr}
\hline DoF & $\mathscr{E}_{\mathrm{SPR}-\mathrm{C}}$ & $\mathscr{E}_{\mathrm{FUB}} \mathrm{SPR}_{\mathrm{SP}}$ & \multicolumn{1}{c}{$\theta_{\mathrm{SPR}-\mathrm{C}}^{\prime}$} & \multicolumn{1}{c}{$\theta_{\mathrm{FUB}}^{\prime}{ }_{\mathrm{SPR}-\mathrm{C}}$} & \multicolumn{1}{c}{$C_{\Omega}^{*}$} \\
\hline 1,050 & 0.1600000 & $1,490.0000000$ & 4.6420880 & $43,453.9979900$ & 1.0000000 \\
1,660 & 0.0691000 & 0.2470000 & 3.4497701 & 12.3470740 & 0.0002120 \\
2,420 & 0.0369000 & 0.1630000 & 2.9581056 & 13.0168171 & 0.0002370 \\
3,700 & 0.0191000 & 0.0862000 & 2.9451810 & 13.2774772 & 0.0002220 \\
5,620 & 0.0084200 & 0.0622000 & 1.7989380 & 13.3058002 & 0.0003450 \\
9,160 & 0.0041400 & 0.0402000 & 1.4372523 & 13.9554529 & 0.0004570 \\
14,600 & 0.0020700 & 0.0085500 & 1.3763781 & 5.6981664 & 0.0001840 \\
23,600 & 0.0010700 & 0.0045700 & 1.2049707 & 5.1223167 & 0.0001730 \\
39,400 & 0.0005610 & 0.0022600 & 1.1430562 & 4.5964216 & 0.0002070 \\
66,600 & 0.0003000 & 0.0006920 & 1.1180716 & 2.5819564 & 0.0001210 \\
119,000 & 0.0001600 & 0.0003580 & 1.1006355 & 2.4612852 & 0.0001090 \\
255,000 & 0.0000727 & 0.0001950 & 1.1221196 & 3.0056961 & 0.0001050 \\
\hline
\end{tabular}

Additionally to these tables, Figure 14 shows the convergence analysis for this problem. We observe that the asymptotic convergence rate is being achieved for 
both, $Q_{1}$ and $Q_{2}$ elements. The upper bound $\hat{\mathscr{E}}_{\mathrm{UB}}$ tends to approximate from above to the error indicator $\mathscr{E}$ which uses the SPR-C technique that is considered asymptotically exact in this situation.
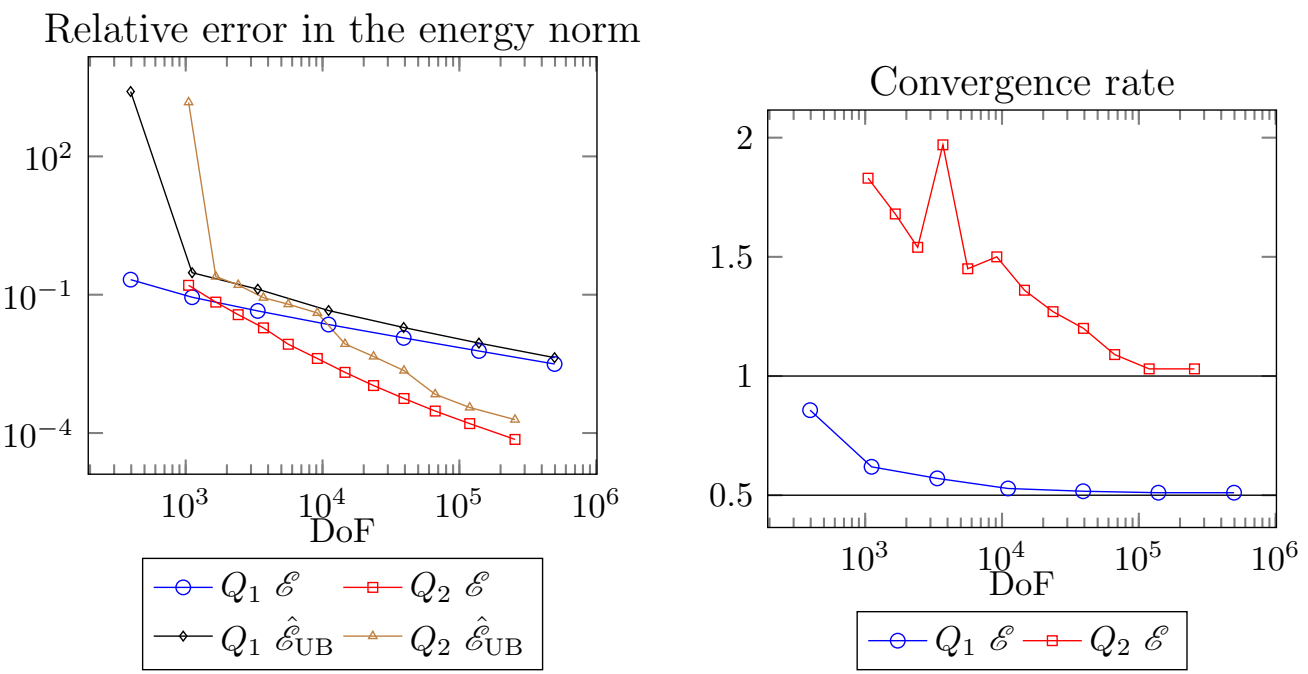

Figure 14: Problem 3. $h$-adaptive refinement. Convergence analysisof the FE solution for bilienar and biquadratic elements. 


\section{Conclusions}

Traditionally, the upper bounding techniques for the error in energy norm were dominated by residual-based error estimation and dual formulations, while the standard recovery-based techniques were unable to provide upper bounds. We have presented a hybrid SPR-based upper bounding method for the linear elasticity problem. This method is a two-step process. In the first step a very accurate and nearly-equilibrated recovery process, the SPR-C technique, is used to provide a non-guaranteed upper bound. In order to guarantee the upper bound property the correction terms introduced in [33] that take into account the equilibrium defaults of the recovered solution have to be taken into consideration. Thus, the second step, which represents the main contribution of the paper, consists of evaluating upper bounds of the correction terms. To bound these terms we use a constant dependent residual explicit-type error estimator. Additionally we also propose an efficient method to numerically evaluate the constant. We have shown that the convergence rate of the bound of the correction terms is higher than that of the error estimator provided by the SPR-C technique, thus concluding that the error estimator, even without the correction terms, would asymptotically tend to the exact error from above. The numerical results presented in section 5 show the accuracy of the proposed upper bounding technique, and also its convergence from above to the exact error. The results presented in this work show this technique as an alternative to traditional bounding methods for practical applications. However the application of the proposed technique, as well as the technique presented in [33], to the problems with singular solutions does not provide accurate results and this is a subject in which we are working in.

\section{Acknowledgements}

This work has been developed within the framework of research project DPI201346317-R of the Ministerio de Economía y Competitividad (Spain). The financial support of the FPU program (AP2008-01086), the funding from Universitat Politècnica de València and Generalitat Valenciana (PROMETEO/2012/023) are also acknowledged.

The authors also thank the support of the Framework Programme 7 Initial Training Network Funding under grant number 289361 "Integrating Numerical Simulation and Geometric Design Technology."

\section{References}

[1] Babuška I, Rheinboldt WC. A-posteriori error estimates for the finite element method. International Journal for Numerical Methods in Engineering 1978; 12(10):1597-1615.

[2] Ainsworth M, Oden JT. A posteriori Error Estimation in Finite Element Analysis, vol. 142. John Wiley \& Sons: Chichester, 2000. 
[3] Díez P, Parés N, Huerta A. Recovering lower bounds of the error by postprocessing implicit residual a posteriori error estimates. International Journal for Numerical Methods in Engineering 2003; 56(10):1465-1488.

[4] Díez P, Parés N, Huerta A. Accurate upper and lower error bounds by solving flux-free local problems in stars. Revue européenne des éléments finis 2004; 13(5-6-7):497.

[5] Gerasimov T, Rüter M, Stein E. An explicit residual-type error estimator for Q 1 -quadrilateral extended finite element method in two-dimensional linear elastic fracture mechanics. International Journal for Numerical Methods in Engineering 2012; 90(April):1118-1155.

[6] Carstensen C, Funken SA. Constants in Clément-Interpolation error and residual-based a posteriori estimates in finite element methods. East-West J. Numer- Math 2000; 8(3):153-175.

[7] Johnson C, Hansbo P. Adaptive finite element methods in computational mechanics. Computer Methods in Applied Mechanics and Engineering 1992; 101:143-181.

[8] Stein E, Gerasimov T, Rüter M. Error-controlled adaptive multiscale analysis for crack initiation and propagation in brittle materials. Adaptive Modeling and Simulation 2013, Moitinho de Almeida JP, Díez P, Tiago C, Parés N (eds.), Lisbon, 2013; 26.

[9] Gerasimov T, Stein E, Wriggers P. Constant-free explicit error estimator with sharp upper error bound property for adaptive FE analysis in elasticity and fracture. International Journal for Numerical Methods in Engineering Oct 2014; :on-line.

[10] Almeida Pereirea OJB, Moitinho de Almeida JP, Maunder EAW. Adaptive methods for hybrid equilibrium finite element models. Computational Methods in Applied Mechanics and Engineering 1999; 176:19-39.

[11] Ladevèze P, Leguillon D. Error estimate procedure in the finite element method and applications. SIAM Journal on Numerical Analysis 1983; 20(3):485-509.

[12] Almeida Pereira OJB, Moitinho de Almeida JP. A posteriori error estimation for equilibrium finite elements in elastostatic problems. Computer Assisted Mechanics and Engineering Sciences 2001; 8(2-3):439-453.

[13] Moitinho de Almeida JP, Maunder EAW. Recovery of equilibrium on star patches using a partition of unity technique. International Journal for $\mathrm{Nu}$ merical Methods in Engineering 2009; 79:1493-1516.

[14] Ladevèze P, Chamoin L, Florentin E. A new non-intrusive technique for the construction of admissible stress fields in model verification. Computer Methods in Applied Mechanics and Engineering 2010; 199(9-12):766-777. 
[15] Ladevèze P, Maunder EAW. A general method for recovering tractions equilibrating element. Computer Methods in Applied Mechanics and Engineering 1996; 137(2):111-151.

[16] Ladevèze P, Rougeot P. New advances on a posteriori error on constitutive relation in fe analysis. Computer Methods in Applied Mechanics and Engineering 1997; 150(1-4):239-249.

[17] Ladevèze P, Rougeot P, Blanchard P, Moreau JP. Local error estimators for finite element linear analysis. Computer Methods in Applied Mechanics and Engineering 1999; 176(1-4):231-246.

[18] Zienkiewicz OC, Zhu JZ. A simple error estimator and adaptive procedure for practical engineering analysis. International Journal for Numerical Methods in Engineering 1987; 24(2):337-357.

[19] Zienkiewicz OC, Zhu JZ. The superconvergent patch recovery and a posteriori error estimates. Part 1: The recovery technique. International Journal for Numerical Methods in Engineering 1992; 33(7):1331-1364.

[20] Zienkiewicz OC, Zhu JZ. The superconvergent patch recovery and a posteriori error estimates. Part 2: Error estimates and adaptivity. International Journal for Numerical Methods in Engineering 1992; 33(7):1365-1382.

[21] Krizek M, Neittaanmäki P. On Superconvergence Techniques. Acta Applicandae Mathematicae 1987; 9:175-198.

[22] Zhang Z, Zhu JZ. Analysis of the superconvergent patch recovery technique and a posteriori error estimator in the finite element method (I). Computer Methods in Applied Mechanics and Engineering 1995; 163(1-4):159-170.

[23] Rodriguez R. Some Remarks on Zienkiewicz-Zhu Estimator. Numerical Methods for Partial Differential Equations 1994; 635(10):625-635.

[24] Durán R, Muschietti MA, Rodriguez R. On the asymptotic exactness of error estimators for linear triangular finite elements. Numerische Mathematik 1991; 59:107-127.

[25] Wiberg NE, Abdulwahab F, Ziukas S. Enhanced superconvergent patch recovery incorporating equilibrium and boundary conditions. International Journal for Numerical Methods in Engineering 1994; 37(20):3417-3440.

[26] Blacker T, Belytschko T. Superconvergent patch recovery with equilibrium and conjoint interpolant enhancements. International Journal for Numerical Methods in Engineering 1994; 37(3):517-536.

[27] Ramsay ACA, Maunder EAW. Effective error estimation from continuous, boundary admissible estimated stress fields. Computers \& Structures 1996; 61(2):331-343. 
[28] Ródenas JJ, Tur M, Fuenmayor FJ, Vercher A. Improvement of the superconvergent patch recovery technique by the use of constraint equations: the SPR-C technique. International Journal for Numerical Methods in Engineering 2007; 70(6):705-727.

[29] Ródenas JJ, Giner E, Fuenmayor FJ, González-Estrada OA. Accurate recoverytype error estimation for linear elastic fracture mechanics in FEM and X-FEM based on a singular+smooth field splitting. International Conference on Adaptive Modeling and Simulation. ADMOS 2007, International Center for Numerical Methods in Engineering (CIMNE), 2007; 202-205.

[30] Ródenas JJ, González-Estrada OA, Tarancón JE, Fuenmayor FJ. A recoverytype error estimator for the extended finite element method based on singular+smooth stress field splitting. International Journal for Numerical Methods in Engineering 2008; 76(4):545-571.

[31] Carstensen C, Funken Sa. Averaging technique for FE - a posteriori error control in elasticity. Part I: Conforming FEM. Computer Methods in Applied Mechanics and Engineering Jan 2001; 190(18-19):2483-2498.

[32] Carstensen C, Funken SA. Averaging technique for FE - a posteriori error control in elasticity . Part II : $\lambda$-independent estimates. Computational Methods in Applied Mechanics and Engineering 2001; 190(190):4663-4675.

[33] Díez P, Ródenas JJ, Zienkiewicz OC. Equilibrated patch recovery error estimates : simple and accurate upper bounds of the error. International Journal for Numerical Methods in Engineering 2007; 69(August 200610):2075-2098.

[34] Ródenas JJ, González-Estrada OA, Díez P, Fuenmayor FJ. Accurate recoverybased upper error bounds for the extended finite element framework. Computer Methods in Applied Mechanics and Engineering 2010; 199(37-40):2607-2621.

[35] Nadal E, Bordas S, Ródenas JJ, Tarancón JE, Tur M. Accurate Stress Recovery for the Two-Dimensional Fixed Grid Finite Element Method. Procedings of the Tenth International Conference on Computational Structures Technology, 2010; $1-20$.

[36] Nadal E, Ródenas JJ, Albelda J, Tur M, Tarancón JE, Fuenmayor FJ. Efficient Finite Element Methodology Based on Cartesian Grids: Application to Structural Shape Optimization. Abstract and Applied Analysis 2013; :1-19.

[37] Nadal E. Cartesian grid FEM (cgFEM): High performance h-adaptive FE analysis with efficient error control. Application to structural shape optimization. PhD Thesis, Universitat Politècnica de València 2014.

[38] Bishop J. Rapid stress analysis of geometrically complex domains using implicit meshing. Computational Mechanics 2003; 30:460-478.

[39] Xiao QZ, Karihaloo BL. Improving the accuracy of XFEM crack tip fields using higher order quadrature and statically admissible stress recovery. International Journal for Numerical Methods in Engineering 2006; 66(9):1378-1410. 
[40] Bordas SPA, Duflot M. Derivative recovery and a posteriori error estimate for extended finite elements. Computer Methods in Applied Mechanics and Engineering 2007; 196(35-36):3381-3399.

[41] Bordas SPA, Duflot M, Le P. A simple error estimator for extended finite elements. Communications in Numerical Methods in Engineering 2008; 24(11):961-971.

[42] Duflot M, Bordas SPA. A posteriori error estimation for extended finite elements by an extended global recovery. International Journal for Numerical Methods in Engineering 2008; 76:1123-1138.

[43] González-Estrada OA, Ródenas JJ, Fuenmayor FJ, Chinesta F. Exact satisfaction of boundary equilibrium to obtain upper error bounds in energy norm using MLS recovery techniques. Adaptive Modeling and Simulation. Proceedings of V ADMOS 2011, Aubry D, Díez P, Tie B, Parés N (eds.), CINME: Paris, 2011.

[44] Zienkiewicz OC, Taylor R. The Finite Element Method, vol. 1. 4 edn., Butterworth-Heinemann: Oxford, UK, 1989.

[45] Wiberg NE, Abdulwahab F. Patch recovery based on superconvergent derivatives and equilibrium. International Journal for Numerical Methods in Engineering 1993; 36(16):2703-2724.

[46] Aalto J. Built-in field equations for recovery procedures. Computers $\&$ Structures 1997; 64(1-4):157-176.

[47] Aalto J, Isoherranen H. An element by element recovery method with built-in field equations. Computers \& Structures 1997; 64(1-4):177-196.

[48] Aalto J, Aman M. Polynomial representations for patch recovery procedures. Computers \& Structures 1999; 73(1-5):119-146.

[49] Kvamsdal T, Okstad KM. Error estimation based on Superconvergent Patch Recovery using statically admissible stress fields. International Journal for $\mathrm{Nu}$ merical Methods in Engineering 1998; 42(3):443-472.

[50] González-Estrada OA, Nadal E, Ródenas JJ, Kerfriden P, Bordas SPA, Fuenmayor FJ. Mesh adaptivity driven by goal-oriented locally equilibrated superconvergent patch recovery. Computational Mechanics Oct 2013; .

[51] Ciarlet PG. The finite element method for elliptic problems. 1 edn., NorthHolland publishing company, 1978.

[52] Ainsworth M, Zhu JZ, Craig AW, Zienkiewicz OC. Analysis of the ZienkiewiczZhu a-posteriori error estimator in the finite element method. International Journal for Numerical Methods in Engineering 1989; 28(9):2161-2174.

[53] Rüter M, Gerasimov T, Stein E. Goal-oriented explicit residual-type error estimates in XFEM. Computational Mechanics 2012; 52(2):361-376. 